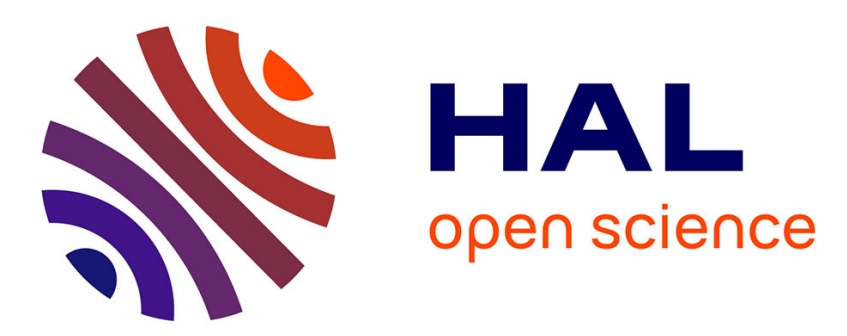

\title{
Critical crossover functions for simple fluids: towards the crossover modelling uniqueness
}

Yves Garrabos, Carole Lecoutre, Samuel Marre, Bernard Le Neindre, Inseob Hahn

\section{- To cite this version:}

Yves Garrabos, Carole Lecoutre, Samuel Marre, Bernard Le Neindre, Inseob Hahn. Critical crossover functions for simple fluids: towards the crossover modelling uniqueness. Journal of Statistical Physics, 2016, 165 (3), pp.471-506. 10.1007/s10955-016-1627-4 . hal-01389620

\section{HAL Id: hal-01389620 \\ https://hal.science/hal-01389620}

Submitted on 20 Jan 2021

HAL is a multi-disciplinary open access archive for the deposit and dissemination of scientific research documents, whether they are published or not. The documents may come from teaching and research institutions in France or abroad, or from public or private research centers.
L'archive ouverte pluridisciplinaire HAL, est destinée au dépôt et à la diffusion de documents scientifiques de niveau recherche, publiés ou non, émanant des établissements d'enseignement et de recherche français ou étrangers, des laboratoires publics ou privés. 


\title{
Critical Crossover Functions for Simple Fluids: Towards the Crossover Modelling Uniqueness
}

\author{
Yves Garrabos ${ }^{1,2}$. Carole Lecoutre ${ }^{1,2}$. \\ Samuel Marre ${ }^{1,2}$. Bernard LeNeindre ${ }^{3,4}$. Inseob Hahn ${ }^{5}$
}

\begin{abstract}
Based on a single non-universal temperature scaling factor present in a simple fluid case, a detailed analysis of non-universal parameters involved in different critical-to-classical crossover models is given. For the infinite limit of the cutoff wave number, a set of three scaling-parameters is defined for each model such that it shows all the shapes of the theoretical crossover functions overlap on the mean crossover function shapes close to the non-trivial fixed point. The analysis of corresponding links between their fluid-dependent parameters opens a route to define a parametric model of crossover equation-of-state, closely satisfying the universal features calculated from the Ising-like limit in the massive renormalization scheme.
\end{abstract}

Keywords Critical crossover functions · Crossover models · Crossover Equation of State · Critical crossover uniqueness $\cdot$ Xenon

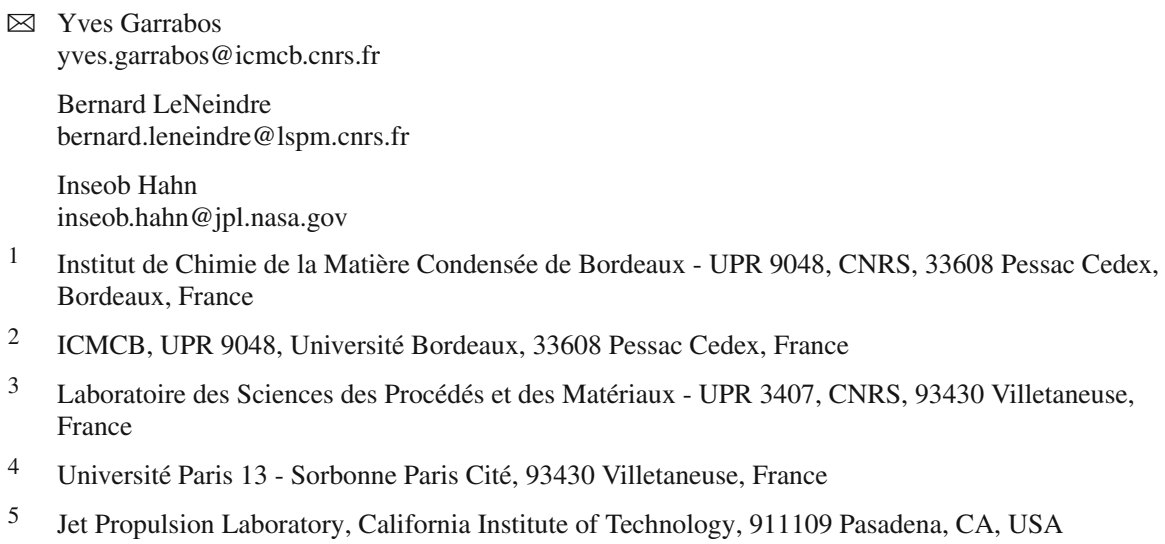




\section{Introduction}

In our previous paper [1], the universal features of one-component fluids close to their vapor-liquid critical point have been analyzed using the theoretical dimensionless crossover functions $F_{P \text {,th }}(t, h=0)$, where $t$ is the theoretical temperature-like field, and $h=0$ is the zero value of the theoretical external ordering (magnetic-like) field. These functions are calculated from the massive renormalization (MR) scheme along the renormalized trajectory which joins the Gaussian fixed point to the non-trivial (so-called Wilson-Fisher) fixed point $[2,3]$. Despite the fact that the experimental data to support this theoretical analysis are obtained at finite distance from the critical temperature, the asymptotic singular behavior of the simple fluids are predicted in conformity with the singular universality estimated within the so-called Ising-like preasymptotic domain (PAD), close to vicinity of the non-trivial fixed point of the $\left(\phi^{2}\right)_{d=3}^{2}(n=1)$ model [4,5] ( $d$ and $n$ are the dimensions of the space and the order parameter density, respectively). For this well-defined Ising-like critical limit along the renormalized trajectory, the universal features are only related to the contributions of two relevant scaling fields and a single irrelevant scaling field [6,7]. As a noticeable result, the universal leading singular behavior of the mean crossover functions [2] are governed by the universal values of two independent \{amplitude-exponent $\}$ pairs while the universal contribution of the confluent corrections-to-scaling is governed by the universal values of a single \{amplitude-lowest exponent\} pair, where the universal central values of the exponents [8] are well controlled.

In such a well-defined theoretical critical limit, each one-component fluid (labelled $f$ ) belonging to the subclass of the simple fluids (labelled $\{1 f\}$ ) is characterized by only two leading physical amplitudes and one first-order confluent amplitude. This non-universal threeamplitude characterization of the one-component fluid is well defined only introducing three non-universal parameters in the mean crossover functions under the form of two dimensionless characteristic scale factors (noted $\vartheta$ and $\psi_{\rho}$ ) and a single coupling constant (noted $g_{0}$ ) of convenient wavelength dimension at $d=3$.

In Ref. [1], the determination of $\vartheta, \psi_{\rho}$, and $g_{0}$ is made de facto without any adjustable parameter when the four critical coordinates of the fluid (xenon as a typical example) are known, taking advantage of the Ising-like master singular behavior of the one-component fluid subclass described by the master crossover functions defined in Ref. [9]. Therefore, the prediction of the Ising-like PAD description only requires the knowledge of the location of the vapor-liquid critical point in the experimental phase surface of $f$, as initially postulated in Refs. [10-12] and shown in a detailed manner in Ref. [13] for the xenon case.

A correlative question arises: how any Ising-like fluid can be characterized with only three amplitudes with different crossover models considering the possibility of finite or infinite nature of the cutoff wave number of the fluid? Indeed, it was noticeable for example that xenon is a simple fluid for which the fitting results of the interpolated asymptotic results for the PAD description are similar when the different crossover models are used in the fitting of the data at finite distance from $T_{c}$. This noticeable asymptotical similarity is here illustrated in Table 1 from the accurate xenon measurements $[14,15]$ of the dimensionless isothermal compressibility $\kappa_{T \text {,expt }}^{*}\left(\Delta \tau^{*}\right)$ above $T_{c}$ and the reduced coexisting density difference $\Delta \tilde{\rho}_{L V \text {,expt }}\left(\left|\Delta \tau^{*}\right|\right)$ below $T_{c}$, already analyzed in Ref. [1] at finite distance from $T_{c}$. Here $\Delta \tau^{*}=\frac{T-T_{C}}{T_{c}}$ is the reduced temperature distance, where $T$ is the temperature and $T_{C}$ the critical temperature. $\Delta \tilde{\rho}_{L V \text {,expt }}=\frac{\rho_{L}-\rho_{V}}{2 \rho_{c}}$, where $\rho_{L}$ and $\rho_{V}$ are the liquid and vapor densities of the coexisting phases below $T_{c}$. In Table 1 are reported the results for the twoterm equations $\kappa_{T, \text { expt }}^{*}\left(\Delta \tau^{*}\right)=\Gamma^{+}\left(\Delta \tau^{*}\right)^{-\gamma}\left[1+a_{\chi}^{+}\left(\Delta \tau^{*}\right)^{\Delta}\right]$ [see Eq. (11) below] and 
$\Delta \tilde{\rho}_{L V, \text { expt }}\left(\left|\Delta \tau^{*}\right|\right)=B\left|\Delta \tau^{*}\right|^{\beta}\left[1+a_{M}\left|\Delta \tau^{*}\right|^{\Delta}\right]$ [see Eq. (12) below] provided by several crossover models labelled MR6 [16-18], MSR [19-22], CLM-I to IV [23-26], CPM [27,28], TGN [29-31], CMM [13] and MR7 [2,3,9]. All these models are succinctly presented below in Sect. 3.1. We note that the distinction between the initial (noted MR6 [16]) and upgraded (noted MR7 [2]) crossover functions calculated from the MR scheme is only made in lines 1 and 7 of Table 1, while the following discussion always refers to the upgraded mean crossover functions (noted MR) given in Ref. [3]. Despite some missing data (explained in Appendix 1), Table 1 underlines the remarkable similitude of the interpolated amplitude values for both xenon properties above and below $T_{c}$.

However, as discussed in detail in Refs. $[1,13]$, the accurate xenon measurements of interest $[14,15]$ were performed in finite temperature ranges well beyond the PAD, i.e., in the temperature ranges where the above restricted two-term equations are not valid. The theoretical analyses of the corresponding experimental data leads to unavoidable difficulties concerning the number and the nature of the fluid-dependent parameters introduced in the data fitting from the different critical-to-classical crossover modelling, specially concerning the contribution of higher-order confluent corrections-to-scaling due to additional irrelevant fields. This nonasymptotic fitting situation is briefly summarized in Appendix 1 for the case of xenon data. Therefore, the fact that the different crossover functions used at a finite distance from $T_{c}$ provide comparable estimates of the interpolated central values of the amplitudes $\Gamma^{+}, B, a_{\chi}^{+}$, and $a_{M}$, still remains as a question to understand in a detailed manner.

Indeed, the origin of the universal nature of the non-similarity can be attributed to the different Ising-like limits used in each model, i.e., the small differences between the values of the universal critical exponents and the universal combinations and ratios between the amplitudes used in each model. This universal non-similarity addresses the comparison between the three-universal amplitudes which characterize each model to the ones characterizing the MR functions. However, the origin of the other additional non-universal nature of the nonsimilarity is more complex to understand as it is related to the exact number and nature of the fluid-dependent parameters introduced in each model. When only three non-universal parameters are used in a crossover model where the well-defined Ising-like limit is characterized by only three-universal amplitudes, we expect a quasi-exact similarity with the MR scheme, as suggested in Ref. [1] and demonstrated below. Such a three-parameter model is refered in the following as a model with infinite cutoff wave number.

The main concern of the present work is to understand the universal asymptotic similarity of the crossover models before to analyze the non-universal similarity of the intrinsic free parameters involved of each model. Our understanding of the universal similarity of the crossover models takes the same approach to calculate the master crossover functions of Ref. [9], which are similar to the MR mean crossover functions of Ref. [3], introducing three universal scale factors. This approach thus ignores the minor differences in the universal features (universal values of the critical exponent and the amplitude combinations) estimated from each model.

The next step distinguishes the two cases of a model with only three non-universal parameters and with more than three non-universal parameters. As previously discussed in Appendix 1 of Ref. [9], the similarity of the confluent asymptotic shapes occurs for both cases, when the Ising-like critical crossover regime is governed by a single non-universal quantity associated with the contribution of a single irrelevant field along the renormalized trajectory. Therefore, our attention is focussed on the reduction by one of the exact number of non-universal parameters, mainly noting that a universal link can be defined theoretically between the temperature-like Ginzburg number $G$ [32] and the first leading confluent 
amplitudes $a_{\chi}^{+}$and $a_{M}$ in the case of infinite cutoff wave number, as expected from the renormalization group scheme [5].

Finally, the present work addresses the knowledge of two, readily independent, nonuniversal asymptotic parameters, which characterize the Ising-like critical behavior of any simple fluid when $T \rightarrow T_{c}$. Such an asymptotic behavior is thus in conformity with the two-scale-factor universality associated with the two relevant fields crossing the non-trivial critical point. As a result, in any selected model, the simple fluid singular behavior close to their liquid-vapor critical point can be modelled in conformity with the MR scheme. This modelling approach opens the route to construct a crossover master model of the parametric equation-of-state which matches the universal features estimated from the MR functions.

The paper is organized as follows. Section 2 summarizes the fluid description using the mean crossover functions estimated from the massive renormalization scheme. Using the definitions and notations of Ref. [1], this section describes the three-universal amplitude characterization of the universal MR-PAD description and the three-nonuniversal parameter characterization of the fluid-PAD description. Section 3 recalls descriptions using several crossover models provided from different theoretical approaches. A special attention is given to the true origin of the three-amplitude similarity observed in the universal model-PAD characterization regardless of the theoretical schemes used to construct the crossover model. Section 4 provides the basic understanding of this universal model-PAD similarity for the limit of infinite cutoff wavenumber. Section 5 confirms the uniqueness of the fluid PAD description from the different crossover models when a single crossover parameter is needed to characterize the fluid singular behavior at finite distance from $T_{c}$. The equations linking their fluid-dependent parameters are provided, focussing our discussion on the minimum number (three) of free parameters and their respective (leading prefactors or crossover) Isinglike nature. Conclusion is given in Sect. 6 which opens a route to construct a parametric crossover model where the universal features of the Ising-like limit are similar to the ones estimated from the massive renormalization scheme. Appendix 1 gives a brief review of the fitting conditions of the xenon singular behaviors which provide the results reported in Table 1. Appendix 2 provides the equations which demonstrate the asymptotic two-scalefactor similarity of all the crossover models for the limit of infinite cutoff wavenumber, in order to satisfy the phenomenological Ising-like characterization of each one-component fluid from the four generalized critical coordinates of its liquid-vapor critical point.

\section{Summarized Fluid Characterization Using the MR Mean Crossover Functions}

\subsection{Ising-Like Universal Limit of the MR Crossover Functions}

As expected from the renormalization group scheme, the well-defined non-trivial limit calculated in Ref. [2] corresponds to the one obtained from the standard Landau-Ginzburg Hamiltonian [4,5], where $r-r_{c}$ is the distance to the critical point and $u$ is the coupling constant that controls the interaction scale (with notations of Ref. [5]). The relevant parameters are the temperature-like Ginzburg number $G=u^{\frac{2}{4-d}}$ [32], which scales the reduced temperature (such as $t \equiv \frac{\left|\Delta \tau^{*}\right|}{G}$ ), and its magnetic-like counterpart $G_{h}=u^{\frac{d+2}{2(4-d)}}$ [33], which scales the ordering field (such as $h=\frac{\Delta \tilde{\mu}}{G_{h}}$ ). $\Delta \tau^{*}$ and $\Delta \tilde{\mu}$ are the physical temperature and ordering fields, respectively (see below). The standard critical behavior corresponds to $\left|\Delta \tau^{*}\right| \equiv r-r_{c} \ll G$ and $\Delta \tilde{\mu} \ll G_{h}$. Considering the limit $u \rightarrow 0,\left(r-r_{c}\right) \rightarrow 0$, with 


\section{C}

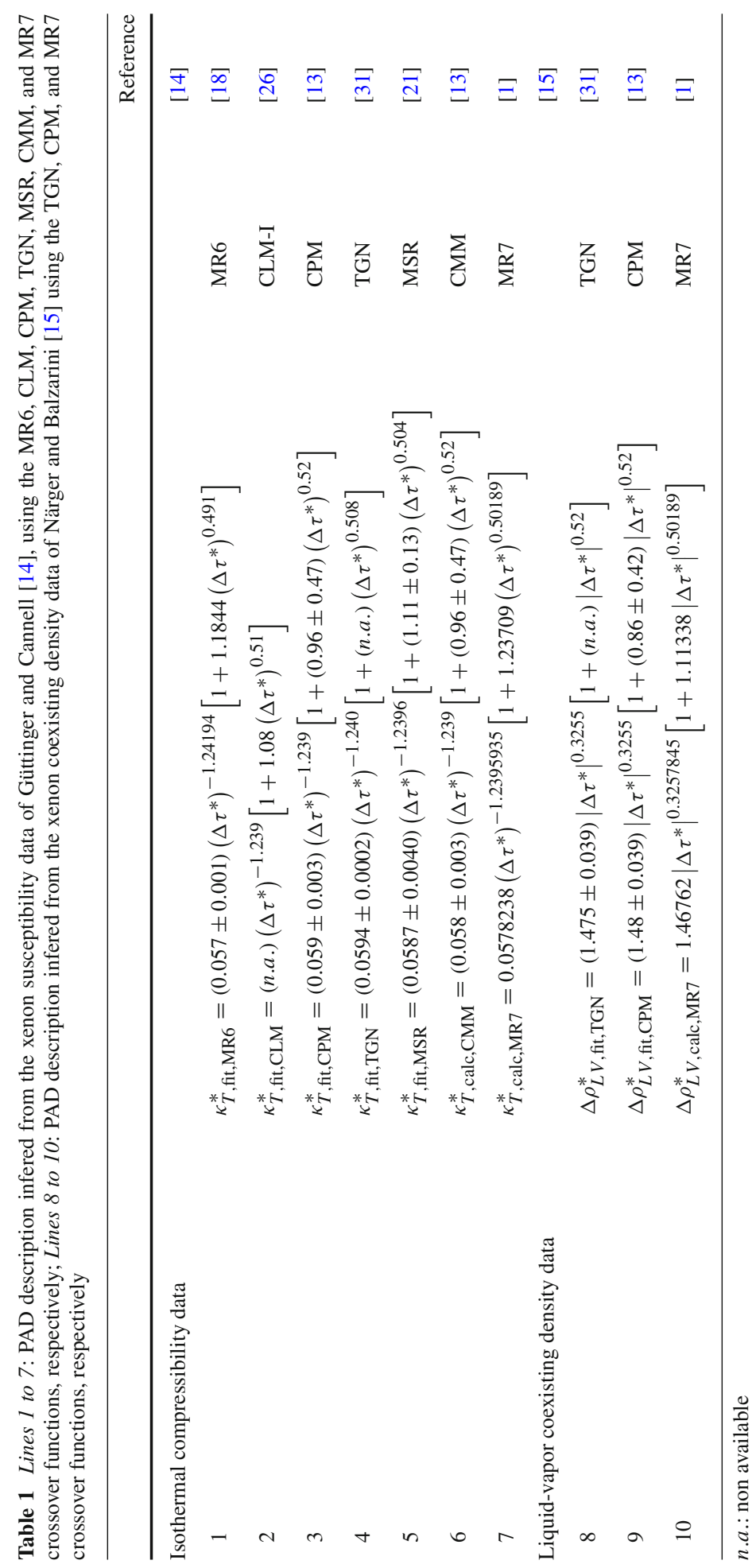


$\frac{\left|\Delta \tau^{*}\right|}{G}$ fixed, provides accurate theoretical computations of the universal functions, such as $F_{P, \text { th }}(t)$ estimated in Refs. [2,3] and used in Ref. [1]. This critical crossover limit is universal for any similar Hamiltonians as long as the interaction is short-ranged.

For a better understanding of this well-defined critical limit, each infinite Wegner-like expansion [34] resummed as a form of the mean theoretical function $F_{P \text {,th }}$ [3] can be approximated by the following two-term forms valid within the PAD, i.e., restricted to the singular contributions of the leading asymptotic term and the first-order term of the confluent corrections when $t \ll 1$ at $h=0$ [3],

$$
F_{P, \text { th }}(t)=\mathbb{Z}_{P}^{ \pm} t^{-\pi_{P}}\left(1+\mathbb{Z}_{P}^{1, \pm} t^{\Delta}\right)
$$

In Eq. (1), $\mathbb{Z}_{P}^{ \pm}$and $\mathbb{Z}_{P}^{1, \pm}$ are the calculated universal amplitudes of the corresponding mean crossover function. The indices + and - indicate the single, high- and the two, low-temperature phase regions, respectively. $\pi_{P}$ is the universal critical exponent which governs the Isinglike asymptotic singular power law behavior of $F_{P \text {,th }}(t)$ for $t \rightarrow 0 . \Delta=\omega v$ is the universal confluent exponent. $\omega$ is the lowest universal correction exponent related to the scaling contribution of an irrelevant field. $v$ is the universal critical exponent which governs the Ising-like asymptotic power law behavior of the correlation length.

As a remarkable result strictly valid within the PAD, the functions $F_{P, \text { th }}(t)$ are then characterized by only two independent leading amplitudes among the complete $\left\{\mathbb{Z}_{P}^{ \pm}\right\}$universal set and a single confluent amplitude among the complete $\left\{\mathbb{Z}_{P}^{1, \pm}\right\}$ universal set. This universal three-amplitude characterization is due to the universal combinations and ratios between the $\mathbb{Z}_{P}^{ \pm}$amplitudes (so-called two-scale-factor universality induced by the two relevant fields) and to the universal ratios between the $\mathbb{Z}_{P}^{1, \pm}$ amplitudes (the confluent universality induced by the single irrelevant field). The universal three-amplitude characterization of the MR results is thus similar to the universal three-exponent characterization of the theoretical $O$ (1) universality class controlled by the universal scaling laws with only two independent critical exponents (among the complete $\left\{\pi_{P}\right\}$ set), while the single lowest value of the correction exponent $\omega$ accounts for all the confluent crossover contributions.

To illustrate the three-amplitude sets characterizing the Ising-like theoretical universality within the PAD, we have selected here the three mean crossover functions $F_{P \text {,th }}=$ $\left\{\ell_{\mathrm{th}} ; \chi_{\mathrm{th}} ; m_{\mathrm{th}}\right\}$ of Ref. [1], namely, the correlation length $\ell_{\mathrm{th}}(t)$ and the magneticlike susceptibility $\chi_{\text {th }}(t)$ in the one-phase domain, and the magnetizationlike density $m_{t h}(|t|)$ in the two-phase domain. The corresponding Eq. (1) can be written as follows

$$
\begin{aligned}
\ell_{\mathrm{PAD}, \mathrm{th}}(t) & =\left(\mathbb{Z}_{\xi}^{+}\right)^{-1} t^{-v}\left(1+\mathbb{Z}_{\xi}^{1,+} t^{\Delta}\right) \\
\chi_{\mathrm{PAD}, \mathrm{th}}(t) & =\left(\mathbb{Z}_{\chi}^{+}\right)^{-1} t^{-\gamma}\left(1+\mathbb{Z}_{\chi}^{1,+} t^{\Delta}\right) \\
m_{\mathrm{PAD}, \mathrm{th}}(|t|) & =\mathbb{Z}_{M}|t|^{\beta}\left(1+\mathbb{Z}_{M}^{1}|t|^{\Delta}\right)
\end{aligned}
$$

Only considering here $\chi_{\text {th }}$ (with critical exponent $\gamma$ ) and $m_{\text {th }}$ (with critical exponent $\beta$ ) as two independent functions, we can thus define the amplitude set $\left\{\mathbb{Z}_{\chi}^{1,+}=8.56347 ;\left(\mathbb{Z}_{\chi}^{+}\right)^{-1}\right.$ $\left.=0.269571 ; \mathbb{Z}_{M}=0.937528\right\}$ given in Ref. [3] as being the characteristic threeamplitude set of the MR-PAD description. This set is strictly Ising-like equivalent to the set $\left\{\mathbb{Z}_{\chi}^{1,+} ;\left(\mathbb{Z}_{\chi}^{+}\right)^{-1} ;\left(\mathbb{Z}_{\xi}^{+}\right)^{-1}=0.471474\right\}$ selected in Ref. [1], through the $\ell_{\text {th }}(t)$ to $m_{\text {th }}(|t|)$ 
exchange for $h=0$. For both sets, the asymptotic critical crossover provided by the MR mean crossover functions close to the non-trivial fixed point have the same well-defined Ising-like limit.

\subsection{Ising-Like Fluid Characterization Using the MR Crossover Functions}

The singular behaviors $P_{s \text {,expt }}^{*}\left(\left|\Delta \tau^{*}\right|\right)$ of the fluid properties along the critical isochore can be predicted using the following rescaled forms of the mean theoretical functions calculated in Ref. [3]

$$
P_{s, \text { expt }}^{*}\left(\left|\Delta \tau^{*}\right|, \Delta \tilde{\mu}=0\right)=P_{r} F_{P, \text { th }}\left(\vartheta\left|\Delta \tau^{*}\right|, \psi_{\rho} \Delta \tilde{\mu}=0\right)
$$

In Eq. (5), the non-universal nature of each one component fluid can be unambiguously characterized by the three-scale factor set $\left\{\vartheta ; \psi_{\rho} ; g_{0}\right\}$, introduced through the following linearized asymptotic equations:

$$
\begin{aligned}
t & =\vartheta \Delta \tau^{*} \\
h & =\psi_{\rho} \Delta \tilde{\mu} \\
\ell_{\mathrm{th}}(t) & =g_{0} \xi_{\operatorname{expt}}\left(\Delta \tau^{*}\right)
\end{aligned}
$$

$\vartheta$ is the fluid-dependent scale factor along the critical isochore. The superscript $*$ labels a non-dimensional fluid quantity obtained by using a single energy reference [noted $\left(\beta_{c}\right)^{-1}$ below, see Eq. (21)] and a single length reference [noted $\alpha_{c}$ below, see Eq. (22)] in the physical normalization process of the fluid particle properties. $\psi_{\rho}$ is the fluid-dependent scale factor along the critical isotherm, associated to the intensive fields $h \rightarrow 0$ and $\Delta \tilde{\mu} \rightarrow 0$. $\Delta \tilde{\mu}=\tilde{\mu}-\tilde{\mu}_{c}$ is the ordering field, with $\tilde{\mu}=\frac{\mu_{\rho} \rho_{c}}{p_{c}}\left(\tilde{\mu}_{c}=\frac{\mu_{\rho, c} \rho_{c}}{p_{c}}\right)$. Here, $\rho$ is the mass density and $\mu_{\rho}$ is the chemical potential per mass unit. The subscript $c$ refers to a critical parameter of the fluid, such as the critical pressure $p_{c}$. The subscript $\rho$ refers to a specific (per mass unit) quantity and the decorated indicates a normalized quantity which introduces an additional set of energy and length references, such as for example $\tilde{\rho}=\frac{\rho}{\rho_{c}}$ or $\tilde{\mu}=\frac{\mu_{\rho} \rho_{c}}{p_{c}}$. The third nonuniversal parameter $g_{0}$ has the convenient wavelength dimension at $d=3$ to take the practical role of a finite wavelength cutoff, defined at the vapor-liquid critical point. The inverse wavelength $\left(g_{0}\right)^{-1}$ is the natural microscopic length unit for $\ell_{\text {th }}(t)$ to calculate the dimensional correlation length $\xi_{\exp }\left(\Delta \tau^{*}\right)$, through Eq. (8). In such a case, the susbscript $r$ in the non-universal dimensionless prefactor $P_{r}$ of Eq. (5) recalls that the appropriate non classical powers of $g_{0}$ are thus accounted for through the product $g_{0} \alpha_{c}$, as discussed below.

As a similar remarkable result strictly valid within the PAD, the physical asymptotic singular properties $P_{s, \text { expt }}^{*}$ can then be estimated from Eq. (5) as the following restricted two-term forms [3],

$$
P_{s, \operatorname{expt}}^{*}\left(\left|\Delta \tau^{*}\right|\right)=\Pi_{P}^{ \pm}\left(\left|\Delta \tau^{*}\right|\right)^{-\pi_{P}}\left[1+a_{P}^{ \pm}\left(\left|\Delta \tau^{*}\right|\right)^{\Delta}\right]
$$

The complete sets of the leading amplitudes, such as $\Pi_{P}^{ \pm}=P_{r} \vartheta^{-\pi_{P}} \mathbb{Z}_{P}^{ \pm}$, and the confluent amplitudes, such as $a_{P}^{ \pm}=\vartheta^{\Delta} \mathbb{Z}_{P}^{1, \pm}$, depend on the fluid $f$. In such a fluid PAD description, the calculated non-universal nature of each one-component fluid is characterized by only two leading asymptotic amplitudes among the complete $\left\{\Pi_{P}^{ \pm}\right\}$set and a single confluent amplitude among the complete $\left\{a_{P}^{ \pm}\right\}$set, conforming to the Ising-like universal features estimated from the MR scheme. However, the fluid non-universal characterization needs a particular attention, noting that $F_{P \text {,th }}$ are only dependent on the first nonuniversal scale factor $\vartheta$ turning of Eq. (5). Moreover, the dimensionless prefactors $P_{r}$ can be dependent on 
the second non-universal scale factor $\psi_{\rho}$ where are contributing two fluid-dependent lengths $\left(g_{0}\right)^{-1}$ and $\alpha_{c}$ to account for the extensive nature of the theoretical and physical properties.

As for the above illustration in the theoretical case of Eqs. (2) to (4), now we consider the corresponding two-term expansions for $\Delta \tau^{*} \rightarrow 0$ of the dimensional correlation length $\xi_{\text {expt }}$ and the dimensionless properties $\kappa_{T \text {,expt }}^{*}$ and $\Delta \tilde{\rho}_{L V \text {,expt }}$ mentionned in the introduction, i.e.,

$$
\begin{aligned}
\xi_{\text {expt }}\left(\Delta \tau^{*}\right) & =\xi_{0}^{+}\left(\Delta \tau^{*}\right)^{-v}\left[1+a_{\xi}^{+}\left(\Delta \tau^{*}\right)^{\Delta}\right] \\
\kappa_{T, \text { expt }}^{*}\left(\Delta \tau^{*}\right) & =\Gamma^{+}\left(\Delta \tau^{*}\right)^{-\gamma}\left[1+a_{\chi}^{+}\left(\Delta \tau^{*}\right)^{\Delta}\right] \\
\Delta \tilde{\rho}_{L V, \text { expt }}\left(\left|\Delta \tau^{*}\right|\right) & =B\left|\Delta \tau^{*}\right|^{\beta}\left[1+a_{M}\left|\Delta \tau^{*}\right|^{\Delta}\right]
\end{aligned}
$$

We note that $\xi_{0}^{+} \sim$ [length] is mandatory a fluid characteristic length in Eq. (10), which leads to define the physical dimensionless amplitude $\xi^{+}=\frac{\xi_{0}^{+}}{\alpha_{c}}$ from reference to the physical length unit $\alpha_{c}$. Thus $\xi^{+}$must be controlled in terms of the non-universal parameters of each fluid, where both Eqs. (11) and (12), introduce $\left\{a_{\chi}^{+} ; \Gamma^{+} ; B\right\}$ as being the characteristic threeamplitude set to be similar to the three-scale factor set $\left\{\vartheta ; \psi_{\rho} ; g_{0}\right\}$ to define the fluid PAD description. From the term to term matching of the corresponding rescaled Eq. (5), it is easy to obtain the unequivocal relations

$$
\begin{aligned}
a_{\chi}^{+} & =\mathbb{Z}_{\chi}^{1,+} \vartheta^{\Delta} \\
\Gamma^{+} & =\left(\mathbb{Z}_{\chi}^{+}\right)^{-1} \chi_{r}^{+} \vartheta^{-\gamma} \\
B & =\mathbb{Z}_{M} M_{r} \vartheta^{\beta}
\end{aligned}
$$

The prefactor terms $P_{r}$ of the leading amplitudes $\Gamma^{+}$and $B$ are $\chi_{r}^{+}=\left(\mathbb{L}^{\{1 f\}}\right)^{d}\left(\psi_{\rho}\right)^{2}$ and $M_{r}=\left(\mathbb{L}^{\{1 f\}}\right)^{d} \psi_{\rho}$, respectively, where is thus introduced the dimensionless coupling-like constant $\mathbb{L}^{\{1 f\}}$ defined by

$$
\mathbb{L}^{\{1 f\}}=g_{0} \alpha_{c}
$$

From Eq. (8), we also obtain $\xi_{0}^{+}=\left(g_{0}\right)^{-1}\left(\mathbb{Z}_{\xi}^{+}\right)^{-1} \vartheta^{-v}$, which can be rewritten as

$$
\xi^{+}=\left(\mathbb{Z}_{\xi}^{+}\right)^{-1} \xi_{r}^{+} \vartheta^{-v}
$$

with the prefactor $\xi_{r}^{+}=\left(\mathbb{L}^{\{1 f\}}\right)^{-1}$. Therefore, only considering the description of the $\{1 f\}$ subclass of the simple fluid where $\mathbb{L}^{\{1 f\}}=g_{0} \alpha_{c}$ is a master constant, Eq. (16) provides the uniqueness of the length unit used in the renormalized bare quantities and dimensionless physical quantities. As expected in our master PAD description [9], the introduction of the master constant $\mathbb{L}^{\{1 f\}}$ ensures the amplitude combinations $\left(\xi_{r}^{+}\right)^{d} \frac{\left(M_{r}\right)^{2}}{\chi_{r}^{+}}=1$ and $Q_{c}=$ $\left(\xi^{+}\right)^{d} \frac{B^{2}}{\Gamma^{+}}=\left(\mathbb{Z}_{\xi}^{+}\right)^{-d}\left(\mathbb{Z}_{M}\right)^{2} \mathbb{Z}_{\chi}^{+}$to be conform with the two-scale-factor universality (using the scaling law $d v=2 \beta+\gamma)$.

For a refined understanding of the non-trivial critical limit, it should also be recalled that the bare coupling constant, $\left(g_{0}\right)^{\frac{1}{4-d}}$, defined at the critical point has been substituted to the cutoff wave number, $\Lambda$, to play the role of the wave-vector unit, while the MR mean crossover functions were derived in the massive renormalization scheme [16] using infinite cutoff wave number $(\Lambda \rightarrow \infty)$. Nevertheless, for $d=3$, $g_{0}$ has exactly the dimension of 
$\Lambda$, i.e., the inverse length dimension, which is very convenient to derive dimensionless properties, but not essential. Indeed, for the case of the theoretical (dimensionless) and physical (dimensional) correlation length, $\ell_{t h}$ can naturally be compared with the experimental measurements of $\xi_{\text {expt }}$ via the relation $\ell_{t h}=\left(g_{0}\right)^{\frac{1}{4-d}} \xi_{\text {expt }}$. Therefore, in the MR scheme, $g_{0}$ was generally considered as the third non-universal parameter added to $\vartheta$ and $\psi_{\rho}$ to characterize any physical system belonging to the $O(1)$ universality class. However, the introduction of the critical microscopic length, $\alpha_{c}=\left(\frac{k_{B} T_{c}}{p_{c}}\right)^{\frac{1}{d}}$, as a length unit for the simple fluid case, leads to define the physical dimensionless correlation length as $\xi_{\text {expt }}^{*}=\frac{\xi_{\text {expt }}}{\alpha_{c}}$. As a practical consequence, a master (constant) value of $\mathbb{L}^{\{1 f\}}=25.585$ estimated from Ref. [9] can then be used to characterize the one-component fluid subclass (noted $\{1 f\}$ ), i.e., the subclass of comparable simple fluids where the explicit length scale unit $\alpha_{c}$ takes comparable values when $\alpha_{c}$ is calculated from two intensive critical properties $T_{c}$ and $p_{c}$ [see below Eq. (22)]. The length dimensions of the hamiltonian bare quantities (such as $r_{0}$ for example, which leads to $\left|\Delta \tau^{*}\right| \sim r_{0}-r_{0 c} \propto\left(g_{0}\right)^{\frac{2}{4-d}}$ and $\left.\Phi_{0} \sim m \propto\left(g_{0}\right)^{\frac{d-2}{2(4-d)}}\right)$, are then implicitely accounted for, since the density variables correctly account for the critical power dimension of $\mathbb{L}^{\{1 f\}}$.

In return, $\mathbb{L}^{\{1 f\}}=25.585$ can be used as a criteria to define the related universal length range, such as $\ell_{t h} \gtrsim(3-4) \mathbb{L}^{\{1 f\}} \simeq 75-100$, where the universal three-amplitude set seems sufficient to characterize the universal critical crossover regime. As a direct consequence, in Ref. [1], this well-defined Ising-like limit and the ad hoc construction of the mean crossover functions are the two essential keys to calculate the precise local values of the scale factor $\vartheta_{\mathcal{L}}^{ \pm}\left(\left|\Delta \tau^{*}\right|\right)$ in the extended preasymptotic domain, de facto beyond the PAD, but still within the Ising-like side of the intermediate region [3]. From the asymptotic identities $\vartheta \equiv \vartheta_{\mathcal{L}}^{ \pm}$, as well the scale factor $\vartheta$ as the prefactors $P_{r}\left(\psi_{\rho}\right)$, take physical meaning at the Ising-like limit $\left|\Delta \tau^{*}\right| \rightarrow 0$. Finally, the non-universal nature of any simple fluid belonging to the $\{1 f\}$ subclass of the $O$ (1) universality class is characterized by two parameters $\vartheta$ and $\psi_{\rho}$, while the inverse $\vartheta^{-1}$ and $\left(\psi_{\rho}\right)^{-1}$ act as the effective temperature-like and magnetic-like Ginzburg numbers, respectively.

More essential to understand the physical crossover behavior in simple fluids, $\alpha_{c}$ also measures the range of the fluid microscopic interactions, i.e., a key parameter for distinguishing the critical and classical limiting regimes. $\alpha_{c}$ can take physical meaning analyzing the crossover competition between long-ranged critical fluctuations and short-ranged microscopic interactions [1], then using the following equation

$$
\frac{\ell_{\mathrm{th}}(t)}{\mathbb{L}^{\{1 f\}}}=\frac{\xi_{\exp }\left(\Delta \tau^{*}\right)}{\alpha_{c}}
$$

where $\ell_{\mathrm{th}}(t) \sim \mathbb{L}^{\{1 f\}}$ corresponds to $\xi_{\exp }\left(\Delta \tau^{*}\right) \sim \alpha_{c}$. As a result, the range $\ell_{\mathrm{th}}(t)<\mathbb{L}^{\{1 f\}}$ has no microscopic physical sense for a fluid belonging to the $\{1 f\}$ subclass with comparable short-ranged molecular interactions. Only the above range $\ell_{t h} \gtrsim(3-4) \mathbb{L}^{\{1 f\}} \simeq 75-100$ confers a well-defined Ising-like nature to the corresponding theoretical temperature-like range $t=\vartheta_{\mathcal{L}}^{ \pm}\left|\Delta \tau^{*}\right|$, then to the physical temperature range where $\xi_{\text {expt }}\left(\left|\Delta \tau^{*}\right|\right) \gtrsim(3-4) \alpha_{c}$, and, finally, to the experimental temperature range where the identities $\vartheta_{\mathcal{L}}^{ \pm} \equiv \vartheta$ using the calculated values of $\vartheta_{\mathcal{L}}^{ \pm}\left(\left|\Delta \tau^{*}\right|\right)$ beyond the PAD are validated. Reference [1] has provided unambiguous non-analytic equations to define the extension and nature of the fluid crossover behavior characterized by this single value of $\vartheta$ along the critical isochore (and $\psi_{\rho}$ along the critical isotherm, subsequently). It is important to point out that the Ising-like scale factor nature of $\vartheta$ was noticeable, despite the initial arbitrary introduction through the asymptotic 
linear relation $t=\vartheta\left|\Delta \tau^{*}\right|$, which only assumes small values of $t$ and $\Delta \tau^{*}$ (with similar induced Ising-like scale factor nature of $\psi_{\rho}$ such as $\left.h=\psi_{\rho} \Delta \tilde{\mu}\right)$.

\subsection{Master Characterization of the Simple Fluid Subclass}

The above Ising-like critical crossover description of the simple fluid is phenomenologically reinforced from the calculated master crossover functions of Ref. [9], which are Ising-like similar to the MR mean crossover functions without any adjustable non-universal parameter. Assuming the existence of the master relations,

$$
\begin{aligned}
\vartheta\left(Y_{c}\right)^{-1} & =\Theta^{\{1 f\}} \\
\psi_{\rho}\left(Z_{c}\right)^{\frac{1}{2}} & =\Psi^{\{1 f\}}
\end{aligned}
$$

where $\Theta^{\{1 f\}}$ and $\Psi^{\{1 f\}}$ are two additional master constants, all the fluid-dependent parameters and amplitudes are thus estimated from the four generalized critical coordinates $Q_{c, a_{\bar{p}}}^{\min }=\left\{T_{c} ; p_{c} ; v_{\bar{p}, c} ; \gamma_{c}^{\prime}\right\}$ of the liquid-gas critical point. $v_{\bar{p}, c}=\frac{m_{\bar{p}}}{\rho_{c}}$ is the critical molecular volume. $m_{\bar{p}}$ is the particle mass. $\gamma_{c}^{\prime}$ is the common critical direction of the saturation pressure line and critical isochoric line at $T_{c}$. Firstly, these four critical coordinates of $f$ are used

(i) to make the thermodynamics properties and the correlation length dimensionless from the following energy and length references

$$
\begin{aligned}
\left(\beta_{c}\right)^{-1} & =k_{B} T_{c} \\
\alpha_{c} & =\left(\frac{k_{B} T_{c}}{p_{c}}\right)^{\frac{1}{d}}
\end{aligned}
$$

and

(ii) to define the following two dimensionless critical numbers

$$
\begin{aligned}
Y_{c} & =\gamma_{c}^{\prime} \frac{T_{c}}{p_{c}}-1 \\
Z_{c} & =\frac{p_{c} m_{\bar{p}}}{\rho_{c} k_{B} T_{c}}
\end{aligned}
$$

$Y_{c}$ of Eq. (23) and $Z_{c}$ of Eq. (24) are the respective characteristic scale factors of the relevant master fields along the critical isochore and the critical isotherm of $f$. Then, the three master constant set $\left\{\Theta^{\{1 f\}}=4.288 \times 10^{-3} ; \mathbb{L}^{\{1 f\}}=25.585 ; \Psi^{\{1 f\}}=1.75505 \times 10^{-4}\right\}$ [9] take physical meaning on the dimensionless thermodynamic properties of the $\left(Z_{c}\right)^{-1}$ particles filling the critical interaction volume of size $\alpha_{c}=\left(\frac{k_{B} T_{c}}{p_{c}}\right)^{\frac{1}{d}}$, with critical mass $\frac{m_{\bar{p}}}{Z_{c}}=\rho_{c}\left(\alpha_{c}\right)^{d}$. That induces the determination of the three master amplitude set $\left\{\mathcal{Z}_{\chi}^{1,+}=0.555 ; \mathcal{Z}_{\chi}^{+}=0.11975 ; \mathcal{Z}_{M}=0.4665\right\}$ [9], which characterizes the master PAD description of the $\{1 f\}$ subclass. Finally, switching our focus to the interpolated two-term expansions of $\kappa_{T, \text { expt }}^{*}$ and $\Delta \tilde{\rho}_{L V \text {,expt }}$ mentionned in the introduction, we obtain

$$
\begin{aligned}
a_{\chi}^{+} & =\mathcal{Z}_{\chi}^{1,+}\left(Y_{c}\right)^{\Delta} \\
\Gamma^{+} & =\mathcal{Z}_{\chi}^{+}\left(Z_{c}\right)^{-1}\left(Y_{c}\right)^{-\gamma} \\
B & =\mathcal{Z}_{M}\left(Z_{c}\right)^{-\frac{1}{2}}\left(Y_{c}\right)^{\beta}
\end{aligned}
$$


The three-amplitude set $\left\{a_{\chi}^{+} ; \Gamma^{+} ; B\right\}$ is well only a function of the two scale factors $Y_{c}$ and $Z_{c}$ of Eqs. (23) and (24), i.e., a PAD description which only needs the knowledge of the critical point location on the fluid phase surface of equation-of-state $\phi_{a_{\bar{p}}}\left\{p, v_{\bar{p}}, T\right\}=0$. The subscript $a_{\bar{p}}$ recall for a normalized thermodynamic description starting from the Helmholtz free energy of the fluid particle.

\section{Summary of Fluid Characterization Using Other Crossover Theoretical Models}

\subsection{Selected Crossover Models}

\subsubsection{Crossover Functions from the Minimal-Subtraction Renormalization Scheme (MSR Model)}

The implicit MSR model [22] was developped in the minimal-subtraction renormalization scheme $[19,20]$. The MSR crossover functions are essentially equivalent to the MR ones, although differs the way to introduce the set $\{u ; a ; \mu\}$ of three non universal parameters (using notations of Ref. [22] and noting that the MSR parameter $u$ cannot be confused with the previous MR (coupling constant) parameter $u$ ). It should be noted for our present concern, that the RG-based crossover expressions in the minimal-subtraction and massive renormalization schemes were derived using an infinite cutoff wave number $(\Lambda \rightarrow \infty)$. The limitations induced by the introduction of such an infinite value are discussed in Refs. [17, 19]. The present work demonstrates the unequivocal similarity of MSR versus MR ignoring the minor differences between their respective Ising-like universal features, i.e., their slightly different universal values of the critical exponents and amplitude combinations, including the ratios of the first-order confluent amplitudes.

\subsubsection{Parametric Models from Crossover Expansions of the Free Energy (CLM-I to IV, CPM, CMM)}

A series of models [25] used the crossover expansions for the Helmholtz free energy based on the renormalization group matching point method [35-37], initially implemented in the so-called crossover Landau models (noted CLM-I to IV) [23,24,38,39], and subsequently upgraded in the crossover formulations (noted CPM) of the phenomenological parametric forms of the pertinent fields involved in the equation of state [27,28]. These models have thus complemented the crossover models of Ref. [40] obtained on the basis of an expansion in terms of $\varepsilon=4-d$, but restricted to the susceptibility and heat capacity above $T_{c}$, as discussed in Ref. [41].

The common main characteristic of CLM and CPM is the introduction of a minima four non-universal parameters to characterize the crossover behavior of the physical system. We have distinguished three versions, noted CLM-I, CLM-II, and CPM, to precise the role of their respective non-universal parameter sets, namely, $\left\{g ; \bar{u} ; a_{0} ; u_{0}\right\},\left\{\Lambda ; \bar{u} ; c_{t} ; c_{\rho}\right\}$, and $\left\{g ; \bar{u} ; \widetilde{m}_{0} ; \widetilde{l}_{0}\right\}$ (for notations, see Refs. [25] and [28]). We already note that two non-universal parameters, namely $g$ and $\bar{u}$, or $\Lambda$ and $\bar{u}$, are explicit to characterize the classical-to-critical crossover behavior. We recall that CLM-I\&II are asymptotic versions and a especial attention is given to CLM-II for which the previous works [42,43] have already demonstrated the similarity with the master PAD description in the analysis of the singular behaviors of six 
simple fluids different from xenon. Additional CPM-MSR comparisons can be found in Refs. $[26,44]$. We also note the recent development of the master form (noted CMM) [13] of CPM, which works without any adjustable non-universal parameter when the critical coordinates of the liquid-vapor critical point of the one-component fluid are known.

For a more precise comparison with the MR scheme, the present work also considers the reduction by one of the number of non-universal parameters involved in the re-named CLM0-I\&II and CPM0 models (adding the label 0), by fixing $\bar{u}=0$ in CLM-I\&II and CPM (see below and Appendix 2.3). Through this condition, these models are also satisfying the infinite limit for the cutoff wave number $(\Lambda \rightarrow \infty)$, with finite values of the product $\bar{u} \Lambda$, leading to the definition of the crossover functions that only depend on $g$, or $\bar{u} \Lambda$.

\subsubsection{Numerical Crossover Calculations from the Ising Lattice Models (TGN)}

The theoretical crossover curves [31] that have been obtained from numerical calculations for Ising lattice models refer to the model noted TGN [29,30], in which the system-dependent parameter was the tunable, Ginzburg-number-like parameter (noted $G_{\mathrm{Xe}}^{ \pm}$in Ref. [31]). The comparison of these numerical results with CLM-II is given in Ref. [45]. The PAD characterization for the numerical TGN case is not reported here, as it appears to be more complex due to the supplementary dependence on the interaction range of the Ising lattice models which can not be related to the microscopic molecular interactions in simple fluids.

\subsection{Ising-Like Crossover Description Within the PAD}

The above brief presentation of the crossover models is mainly focused on the definition and the number of the non-universal parameters involved in each model. However, we can also use the generic label $\mathrm{W}$ to distinguish the definition and the notations of the universal quantities (exponents and amplitudes) similar to the ones used in the MR crossover description. In a first approximation, the small differences in the universal values of the critical exponents and amplitude combinations (including the universal ratios between the first-order confluent amplitudes) can be ignored. This convenient simplification provides a well-defined Ising-like limit of the asymptotic critical crossover close to the non-trivial fixed point of each model, only characterized by a three (one-confluent and two-leading) universal amplitude set. As the fluid description within the PAD can thus be characterized by the three nonuniversal amplitude set $\left\{a_{\chi}^{+} ; \Gamma^{+} ; B\right\}$, the remaining important Ising-like feature of the crossover models is the exact number of the needed (at least three) non-universal parameters involved in the estimation of this amplitude set. Therefore, in the following a model only using three non-universal parameters is noted as $\mathrm{W}$ while a model using more than three non-universal parameters is noted as $\mathrm{W}_{+}$.

In such a situation, the PAD description of the fluid property $P_{\text {expt }}^{*}$ close to $T_{c}$ can always be defined in terms of one among the two following rescaled fitting equations,

$$
\begin{aligned}
& P_{\text {expt }}^{*}\left(\left|\Delta \tau^{*}\right|, \Delta \tilde{\mu}=0\right)=P_{n, \mathrm{~W}} F_{P, \mathrm{~W}}\left(\left|\frac{\Delta \tau^{*}}{\Delta \tau_{\mathrm{W}}^{*}}\right|\right) \\
& P_{\text {expt }}^{*}\left(\left|\Delta \tau^{*}\right|, \Delta \tilde{\mu}=0\right)=P_{0, \mathrm{~W}_{+}}\left|\Delta \tau^{*}\right|^{\pi P_{P}} F_{P, \mathrm{~W}_{+}}\left(\left|\frac{\Delta \tau^{*}}{\Delta \tau_{\mathrm{W}_{+}}^{*}}\right|\right)
\end{aligned}
$$

introducing the non-universal prefactors $P_{n, \mathrm{~W}}$ or $P_{0, \mathrm{~W}_{+}}$and the non-universal temperature scaling factors $\Delta \tau_{\mathrm{W}}^{*}$ or $\Delta \tau_{\mathrm{W}_{+}}^{*}$ of the temperature field to replace the proper non-universal parameters involved in each model. Fitting Eq. (28) valid for the W model appears thus 
similar to Eq. (5). We note however the introduction of the subscript $n$ which can distinguish the needed intrinsic theoretical process to normalize an universal W quantity, especially the correlation length. Indeed, any three parameter model necessarily appears in conformity with the renormalization schemes that satisfy to the infinite cut-off wave number and coupling constant of wavelength dimension, while $\Delta \tau_{\mathrm{W}}^{*}$ is thus the temperature-like Ginzburg number $G$ (see Appendix 2.2). In Eq. (29) valid for the $\mathrm{W}_{+}$model, we underline the explicit leading power law in term of the physical temperature field. This latter equation only involves the physical process to normalize a fluid thermodynamic property, while in the case of physical correlation length of [Length] dimension, the prefactor $\xi_{0, \mathrm{~W}_{+}}$is mandatory a fluid characteristic length.

In such above fitting forms, $F_{P, \mathrm{~W}}\left(t_{\mathrm{W}}\right)$, or $F_{P, \mathrm{~W}_{+}}\left(t_{\mathrm{W}_{+}}\right)$, are universal dimensionless functions only dependent on the rescaled temperature ratios $t_{\mathrm{W}}=\left|\frac{\Delta \tau^{*}}{\Delta \tau_{\mathrm{W}}^{*}}\right|$ or $t_{\mathrm{W}_{+}}=\left|\frac{\Delta \tau^{*}}{\Delta \tau_{\mathrm{W}_{+}}^{*}}\right|$. Equations (28) and (29) provide an easier understanding of the nature of the two fluiddependent parameters used in each fitting equation, despite the fact that $\Delta \tau_{\mathrm{W}}^{*}\left(\right.$ or $\Delta \tau_{\mathrm{W}_{+}}^{*}$ ), as well as $P_{n, \mathrm{~W}}$ (or $P_{0, \mathrm{~W}_{+}}$), were defined as complex combinations of the proper nonuniversal parameters of $\mathrm{W}$ ( or $\mathrm{W}_{+}$), which implicitely include the needed energy and length references.

To describe the PAD for the Ising-like limit $\left|\Delta \tau^{*}\right| \rightarrow 0$, the universal functions $F_{P, \mathrm{~W}}=$ $\left\{\ell_{\mathrm{W}} ; \chi_{\mathrm{W}} ; m_{\mathrm{W}}\right\}$ (or $F_{P, \mathrm{~W}_{+}}=\left\{\ell_{\mathrm{W}_{+}} ; \chi_{\mathrm{W}_{+}} ; m_{\mathrm{W}_{+}}\right\}$) similar to our previous MR Eqs. (2) to (4), can be written asymptotically as follows

$$
\begin{gathered}
\ell_{\mathrm{PAD}, \mathrm{W}}=Z_{\xi, \mathrm{W}}^{+}\left(t_{\mathrm{W}}\right)^{-v}\left(1+g_{\xi, \mathrm{W}}^{1,+}\left(t_{\mathrm{W}}\right)^{\Delta}\right) \\
\chi_{\mathrm{PAD}, \mathrm{W}}=Z_{\chi, \mathrm{W}}^{+}\left(t_{\mathrm{W}}\right)^{-\gamma}\left(1+g_{\chi, \mathrm{W}}^{1,+}\left(t_{\mathrm{W}}\right)^{\Delta}\right) \\
m_{\mathrm{PAD}, \mathrm{W}}=Z_{M, \mathrm{~W}}\left|t_{\mathrm{W}}\right|^{\beta}\left(1+g_{M, \mathrm{~W}}^{1}\left|t_{\mathrm{W}}\right|^{\Delta}\right) \\
\ell_{\mathrm{PAD}, \mathrm{W}_{+}}=Z_{\xi, \mathrm{W}_{+}}^{+}\left(1+g_{\xi, \mathrm{W}_{+}}^{1,+}\left(t_{\mathrm{W}_{+}}\right)^{\Delta}\right) \\
\chi_{\mathrm{PAD}, \mathrm{W}_{+}}=Z_{\chi, \mathrm{W}_{+}}^{+}\left(1+g_{\chi, \mathrm{W}_{+}}^{1,+}\left(t_{\mathrm{W}_{+}}\right)^{\Delta}\right) \\
m_{\mathrm{PAD}, \mathrm{W}_{+}}=Z_{M, \mathrm{~W}_{+}}\left(1+g_{M, \mathrm{~W}_{+}}^{1}\left|t_{\mathrm{W}_{+}}\right|^{\Delta}\right)
\end{gathered}
$$

Selecting $\chi_{\mathrm{W}}$ (or $\chi_{\mathrm{W}_{+}}$) and $m_{\mathrm{W}}$ (or $m_{\mathrm{W}_{+}}$) as two independent functions of present interest, the Ising-like universality within the PAD appears characterized only through the universal values of the three amplitude set $\left\{g_{\chi, \mathrm{W}}^{1,+} ; Z_{\chi, \mathrm{W}}^{+} ; Z_{M, \mathrm{~W}}\right\}$ (or $\left\{g_{\chi, \mathrm{W}_{+}}^{1,+} ; Z_{\chi, \mathrm{W}_{+}}^{+} ; Z_{M, \mathrm{~W}_{+}}\right\}$). These latter sets for $\mathrm{W}\left(\right.$ or $\mathrm{W}_{+}$) are strictly similar to the above set $\left\{\mathbb{Z}_{\chi}^{1,+} ;\left(\mathbb{Z}_{\chi}^{+}\right)^{-1} ; \mathbb{Z}_{M}\right\}$ of the MR PAD description. All these characteristic universal quantities are reported in columns 2 to 5 of Table 2 ignoring the exact number (three or more) of nonuniversal parameters involved in $\mathrm{W}$ and in columns 6 and 7 of Table 2 for the MR case. They are analyzed in a detailed manner below (Sect. 4).

\section{Similar Universal Crossover Shapes Within the PAD}

The comparative analysis of the selected crossover models needs to distinguish the similarity of the models governed by each universal three-amplitude set such as $\left\{g_{\chi}^{1,+} ; Z_{\chi, \mathrm{W}}^{+} ; Z_{M, \mathrm{~W}}\right\}$ given in Table (2), from the similarity of the intrinsic non-universal parameters governed by a number of parameters, which can be greater than three as noticed in previous Sect. 3.1. 
Table 2 Lines 1 to 3: Three universal amplitudes of the dimensionless crossover functions for the susceptibility above $T_{c}$ and the order parameter density below $T_{c}$, which characterize the complete Ising-like PAD shapes for four different models (CLM, CPM, MSR and MR, columns 2 to 4 and 6, respectively)

\begin{tabular}{llllll}
\hline W & CLMI\&II [25] & CPM [28] & MSR [22] & MR [2] & [3]
\end{tabular}

(a) Characteristic parameters

\begin{tabular}{|c|c|c|c|c|c|c|}
\hline 1 & $g_{\chi, \mathrm{W}}^{1,+}$ & 0.610 & 0.590 & 0.545 & $\left|\mathbb{Z}_{\chi}^{1,+}\right|$ & 8.56347 \\
\hline 2 & $Z_{\chi, \mathrm{W}}^{+}$ & 0.871 & 3.38317 & 0.25 & $\left(\mathbb{Z}_{\chi}^{+}\right)^{-1}$ & 0.269571 \\
\hline 3 & $Z_{M, \mathrm{~W}}$ & 2.05 & 3.28613 & 0.6 & $\mathbb{Z}_{M}$ & 0.937528 \\
\hline \multicolumn{7}{|c|}{ b) Additional parameters } \\
\hline 4 & $g_{M, \mathrm{~W}}^{1}$ & 0.531 & 0.529 & 0.425 & $\mathbb{Z}_{M}^{1}$ & 7.70712 \\
\hline 5 & $Z_{C, \mathrm{~W}}^{+}$ & 2.27 & 1.68210 & $\frac{1}{16 \pi}$ & $\mathbb{Z}_{C}^{+}$ & 1.719788 \\
\hline 6 & $Z_{\xi, \mathrm{W}}^{+}$ & 0.428 & 0.474 & 0.6 & $\left(\mathbb{Z}_{\xi}^{+}\right)^{-1}$ & 0.471474 \\
\hline
\end{tabular}

(c) Ising-like universal features

\begin{tabular}{|c|c|c|c|c|c|c|}
\hline 7 & $\Delta$ & 0.52 & 0.52 & 0.502 & & 0.50189 \\
\hline 8 & $\gamma$ & 1.239 & 1.239 & 1.235 & & 1.2395935 \\
\hline 9 & $\beta$ & 0.3255 & 0.3255 & 0.326 & & 0.3257845 \\
\hline 10 & $R_{C}=\alpha Z_{C, \mathrm{~W}}^{+} \frac{Z_{\chi, \mathrm{W}}^{+}}{\left(Z_{M, \mathrm{~W})^{2}}\right.}$ & 0.052 & 0.0580 & 0.0582 & $\frac{\mathbb{Z}_{C}^{+}}{\mathbb{Z}_{\chi}^{+}\left(\mathbb{Z}_{M}\right)^{2}}$ & 0.0574 \\
\hline 11 & $\left(R_{\xi}^{+}\right)^{d}=\alpha Z_{C, \mathrm{~W}}^{+}\left(Z_{\xi, \mathrm{W}}^{+}\right)^{d}$ & 0.0188 & 0.0188 & 0.0206 & $\frac{\mathbb{Z}_{C}^{+}}{\left(\mathbb{Z}_{\xi}^{+}\right)^{d}}$ & 0.0196 \\
\hline 12 & $\left(Q_{c, \mathrm{~W}}\right)^{-1}=\frac{R_{C}}{\left(R_{\xi}^{+}\right)^{d}}$ & 2.64 & 2.95 & 2.82 & $\frac{\left(\mathbb{Z}_{\xi}^{+}\right)^{d}}{\mathbb{Z}_{\chi}^{+}\left(\mathbb{Z}_{M}\right)^{2}}$ & 2.93 \\
\hline 13 & $\frac{g_{M, \mathrm{~W}}^{1,+}}{g_{\chi, \mathrm{W}}^{1,+}}$ & 0.87 & 0.897 & 0.76 & $\frac{\left|\mathbb{Z}_{\chi}^{1,+}\right|}{\mathbb{Z}_{M}^{1}}$ & 0.9 \\
\hline
\end{tabular}

(d) Matching parameters
$14 \Theta_{\mathrm{W}}$
$5.36 \times 10^{-3} \quad 5.02 \times 10^{-3} \quad 4.16 \times 10^{-3}$
15a $\Psi_{M, \mathrm{~W}}$
$7.2 \times 10^{-4}$
$1.17 \times 10^{-3} 2.43 \times 10^{-4}$
$15 \mathrm{~b} \Psi_{\chi, \mathrm{W}}$
$5.4 \times 10^{-4}$
$1.03 \times 10^{-3} 2.49 \times 10^{-4}$
$16 \ell_{n, \mathrm{~W}}$
$\left(v_{0}\right)^{\frac{1}{3}}=\alpha_{c}$
$\left(v_{0}\right)^{\frac{1}{3}}=\alpha_{c} \quad l_{0}=\alpha_{c}$
$\frac{\left(g_{0}\right)^{-1}}{\mathbb{L}\{1 f\}}=\alpha_{c}$

Line 4: First-order confluent universal amplitude of the crossover function for the order parameter density below $T_{c}$. Lines 5 and 6: Leading universal amplitudes for the specific heat and correlation length above $T_{c}$. Lines 7 to 13: universal exponents and universal amplitude combinations characterizing the Ising-like universal features of each model. Lines 14, 15a and 15b: Two scale factors needed to get quasicomplete matching of the Ising-like crossover shapes of CLM, CPM and MSR with the one of MR (lines 15a and 15b are respectively calculated from Eqs. (41) and (42) to illustrate the possible differences due to the Ising-like universal features estimated for each model). Line 16: Physical length unit of the correlation length

However, as any three-parameter crossover model must be in conformity to the limit $\Lambda \rightarrow \infty$, our primary interest is to demonstrate the quasi-perfect universal matching between all the resulting crossover shapes of the PAD description by Eqs. (30) to (35). Hence, using the data reported in Table 2, we can directely infer the similarity between the characteristics universal quantities (defined in column 2) of CLMI\&II (column 3), CPM (column 4), MSR (column 
5) and the ones (defined in column 6) of MR (column 7), assuming that the crossover models $\mathrm{W}$ are such as:

(i) the Ising-like universality is accounted for using a single independent, universal value of the first-order confluent amplitude $\left(g_{\chi}^{1,+}\right)$ and two independent, universal values of the leading amplitudes $\left(Z_{\chi, \mathrm{W}}^{+}, Z_{M, \mathrm{~W}}\right)$. The corresponding values are given in lines 1 to 3 ;

(ii) a single length unit is used to reduce the correlation lengths and the thermodynamic properties of the models and of the simple fluids belonging to the one-component subclass of universality. The corresponding definition is reported in last line 16;

(iii) the universal nature of the models are in conformity with an infinite cutoff wave number and then are accounted for by introducing only two independent asymptotic parameters among the three free parameters of the model. The control of the Ising-like universal features in each model can be performed using the additional values of the first-order confluent amplitude $g_{M, \mathrm{~W}}^{1}$ (from the magnetic-like crossover function) and the leading amplitudes $Z_{C, \mathrm{~W}}^{+}$(from the specific heat crossover function) and $Z_{\xi, \mathrm{W}}^{+}$(from the correlation length crossover function), given in lines 4 to 6 , respectively.

Therefore all the theoretical crossover shapes must be collapsed (quasi-perfectly) on the corresponding MR curves within the Ising-like PAD. Here quasi-perfectly refers to the neglected small differences between the estimated universal Ising-like values of the exponents and the amplitude combinations proper to each model, illustrated from the specific universal quantities reported in lines 7 to 13. This collapse can now be understood in a similar manner to the collapse [9] between the mean and master crossover functions through the use of the master constants $\Theta^{\{1 f\}}$ and $\Psi^{\{1 f\}}$ [see Eqs. (19) and (20)].

The first step needs to introduce two universal master constants $\Theta_{\mathrm{W}}$ and $\Psi_{\mathrm{W}}$, characteristic of the model W, such as

$$
\begin{aligned}
t & =\Theta_{\mathrm{W}} t_{\mathrm{W}} \\
h & =\Psi_{\mathrm{W}} h_{\mathrm{W}} \\
m_{\mathrm{th}} & =\left(\Psi_{\mathrm{W}}\right)^{-1} m_{\mathrm{W}}
\end{aligned}
$$

where the two latter equations result from the conjugated field-density nature of both variables. $t, h, m_{\text {th }}$ and $t_{\mathrm{W}}, h_{\mathrm{W}}, m_{\mathrm{W}}$ are the temperature-like fields, the ordering magnetic-like fields, and the order parameter magnetization-like densities for the MR and W cases, respectively. From the above discussion, it is obvious that $t_{\mathrm{W}}=\left|\frac{\Delta \tau^{*}}{\Delta \tau_{\mathrm{W}}^{*}}\right|$.

The second step uses the hierarchical term-to-term comparison between the respective PAD description by $\mathrm{W}$ and $\mathrm{MR}$, starting with the universal first-order confluent matching and finishing with the universal asymptotical leading matching. Such a comparison needs the implicit use of the non-universal length unit $\ell_{n, \mathrm{~W}}$ of $\mathrm{W}$, similar to $\left(g_{0}\right)^{-1}$ of MR (see last line 16). The application of $\mathrm{W}$ and $\mathrm{MR}$ can then be restricted to the one-component fluid subclass through the master condition

$$
g_{0} \ell_{n, \mathrm{~W}}=\mathbb{L}^{\{1 f\}}
$$

Equation (39), similar to Eq. (16), provides the needed universal identity $\ell_{\mathrm{W}}\left(t_{\mathrm{W}}\right) \equiv$ $\left(\mathbb{L}^{\{1 f\}}\right)^{-1} \ell_{\text {th }}(t)$. 
Finally, selecting as entry data the values reported in lines 1 to 3 , the values of $\Theta_{\mathrm{W}}$ and $\Psi_{\mathrm{W}}$ given in lines 14 and $15 \mathrm{a} \& \mathrm{~b}$ were obtained using the following universal equations

$$
\begin{gathered}
\Theta_{\mathrm{W}}=\left(\frac{g_{\chi, \mathrm{W}}^{1,+}}{\left|\mathbb{Z}_{\chi}^{1,+}\right|}\right)^{\frac{1}{\Delta}} \\
\Psi_{\chi, \mathrm{W}}=\left[Z_{\chi, \mathrm{W}}^{+} \mathbb{Z}_{\chi}^{+}\left(\Theta_{\mathrm{W}}\right)^{\gamma}\left(\mathbb{L}^{\{1 f\}}\right)^{-d}\right]^{\frac{1}{2}} \\
\Psi_{M, \mathrm{~W}}=Z_{M, \mathrm{~W}}\left(\mathbb{Z}_{M}\right)^{-1}\left(\Theta_{\mathrm{W}}\right)^{-\beta}\left(\mathbb{L}^{\{1 f\}}\right)^{-d}
\end{gathered}
$$

In lines $15 \mathrm{a} \& \mathrm{~b}$, the small differences between both estimates of $\Psi_{\mathrm{W}}$ from Eqs. (41) and (42) are indicative of the non-perfect collapse due to the small differences between the universal values of the critical exponents and amplitude combinations (see lines 7 to 13). Similar small differences (not reported here) can be observed on the $\Theta_{\mathrm{W}}$ estimates, using for example the universal equation $\Theta_{\mathrm{W}}=\left(\frac{g_{M, \mathrm{~W}}^{1}}{\mathbb{Z}_{M}^{1}}\right)^{\frac{1}{\Delta}}$ obtained from the first-order confluent matching in the magnetic-like case below $T_{c}$ (see line 4). In this latter case, the differences are due to the distinct values of the universal ratios $g_{M, \mathrm{~W}}^{1,+} / g_{\chi}^{1,+\mathrm{W}}$ and $\left|\mathbb{Z}_{\chi}^{1,+}\right| / \mathbb{Z}_{M}^{1}$ (see line 13). Nevertheless, despite this intrinsic numerical mismatching from the actual theoretical approaches, the collapse of the universal crossover shapes includes the Ising-like universality features at the first-order of the scaling confluent corrections and satisfies to the two-scalefactor universality of the leading singular power laws. For instance, the correlation length asymptotical matching leads to

$$
\left(\mathbb{Z}_{\xi}^{+}\right)^{-1}\left(\Theta_{\mathrm{W}}\right)^{-v}\left(\mathbb{L}^{\{1 f\}}\right)^{-1}=Z_{\xi, \mathrm{W}}^{+}
$$

(see line 6), which can be considered as a universal hyperscaling combination to estimate $\Theta_{\mathrm{W}}$. Indeed, the similar matching for the specific heat leads to $\mathbb{Z}_{C}^{+}\left(\Theta_{\mathrm{W}}\right)^{2-\alpha}\left(\mathbb{L}^{\{1 f\}}\right)^{d}=Z_{C, \mathrm{~W}}^{+}$, while $\left(R_{\xi}^{+}\right)^{d}=\alpha Z_{C, \mathrm{~W}}^{+}\left(Z_{\xi, \mathrm{W}}^{+}\right)^{d}=\mathbb{Z}_{C}^{+}\left(\mathbb{Z}_{\xi}^{+}\right)^{-d}$, see line 11. Equations (40) from firstorder confluent matching and (43) from asymptotical leading matching mean that $\Theta_{\mathrm{W}}$ is necessarily the single scale factor of $t_{\mathrm{W}}$. $\Theta_{\mathrm{W}}$ characterizes the crossover singular behavior of $\mathrm{W}$, as well as the asymptotical leading singular behavior of $\mathrm{W}$, to be in conformity with the MR estimated ones at $h=h_{\mathrm{W}}=0$. In conclusion, the uniqueness of the universal three-parameter modelling within the PAD is well demonstrated from the data of Table 2.

\section{Non-Universal Three-Parameter Matching Within the PAD of a Simple Fluid}

\subsection{Non-Universal Crossover Within the PAD}

The data reported in Table 2 probe the uniqueness of the universal critical behavior within the PAD whatever the selected model. Therefore, to explain the similarity of the results reported in Table 1 for the xenon case, the next step needs to demonstrate the uniqueness of the nonuniversal parametric estimation of the fluid amplitudes regardless the number and the nature of the intrinsic non-universal parameters involved in each model. Indeed, from the rescaled 


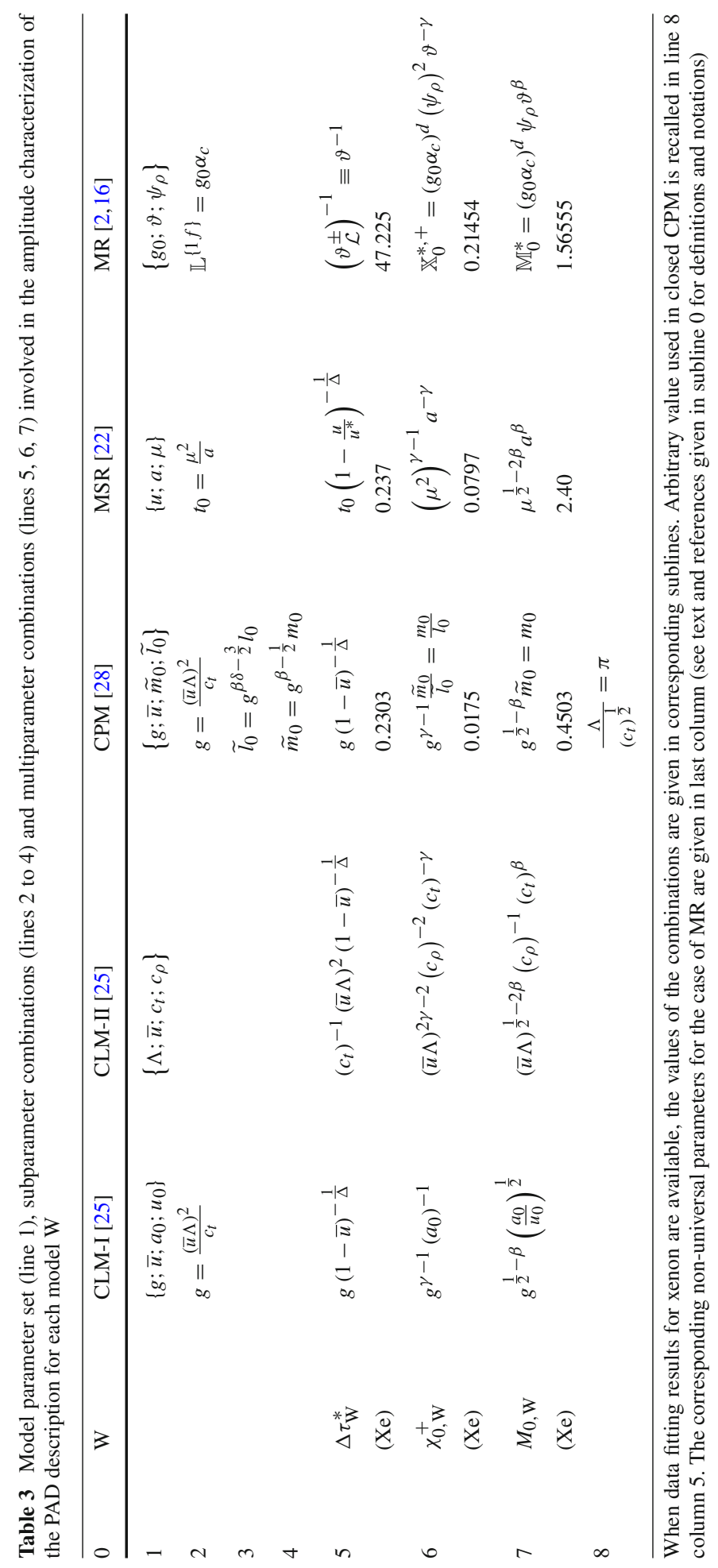


fitting Eqs. (28) and (29), the fluid-dependent amplitudes of the Ising-like characteristic set $\left\{a_{\chi}^{+} ; \Gamma^{+} ; B\right\}$ can be written as follows,

$$
\begin{aligned}
a_{\chi}^{+} & =g_{\chi, \mathrm{W}}^{1,+}\left(\Delta \tau_{\mathrm{W}}^{*}\right)^{-\Delta}=g_{\xi, \mathrm{W}_{+}}^{1,+}\left(\Delta \tau_{\mathrm{W}_{+}}^{*}\right)^{-\Delta} \\
\Gamma^{+} & =Z_{\chi, \mathrm{W}}^{+} \chi_{n, \mathrm{~W}}^{+}\left(\Delta \tau_{\mathrm{W}}^{*}\right)^{\gamma}=Z_{\chi, \mathrm{W}_{+}}^{+} \chi_{0, \mathrm{~W}_{+}}^{+} \\
B & =Z_{M, \mathrm{~W}} M_{n, \mathrm{~W}}\left(\Delta \tau_{\mathrm{W}}^{*}\right)^{-\beta}=Z_{M, \mathrm{~W}_{+}} M_{0, \mathrm{~W}_{+}}
\end{aligned}
$$

The above left equations refer to a $\mathrm{W}$ model while the right equations refer to a $\mathrm{W}_{+}$one. To avoid any reference to the exact number of intrinsic non-universal parameters of $\mathrm{W}$, it is convenient to introduce the leading prefactors $\chi_{0, \mathrm{~W}}^{+}=\chi_{n, \mathrm{~W}}^{+}\left(\Delta \tau_{\mathrm{W}}^{*}\right)^{\gamma}$ and $M_{0, \mathrm{~W}}=M_{n, \mathrm{~W}}\left|\Delta \tau_{\mathrm{W}}^{*}\right|^{-\beta}$ to replace $Z_{\chi, \mathrm{W}}^{+}$and $Z_{M, \mathrm{~W}}$ used in a three-parameter model case. The three-amplitude set $\left\{a_{\chi}^{+} ; \Gamma^{+} ; B\right\}$ can then be exchanged to the three-parameter set $\left\{\Delta \tau_{\mathrm{W}}^{*} ; \chi_{0, \mathrm{~W}}^{+} ; M_{0, \mathrm{~W}}\right\}$. In a similar manner for the MR case, it is also convenient to introduce $\mathbb{X}_{0}^{*,+}=\chi_{r}^{+} \vartheta^{-\gamma}$ and $\mathbb{M}_{0}^{*}=M_{r}|\vartheta|^{\beta}$ to replace $\chi_{r}^{+}=\left(\mathbb{L}^{\{1 f\}}\right)^{d}\left(\psi_{\rho}\right)^{2}$ and $M_{r}=\left(\mathbb{L}^{\{1 f\}}\right)^{d} \psi_{\rho}$, respectively. The three-parameter set $\left\{\vartheta ; \mathbb{X}_{0}^{*,+} ; \mathbb{M}_{0}^{*}\right\}$ replaces the three-scale factor set $\left\{\vartheta ; \psi_{\rho} ; g_{0}\right\}$ and can more directly be compared and matched to $\left\{\Delta \tau_{\mathrm{W}}^{*} ; \chi_{0, \mathrm{~W}}^{+} ; M_{0, \mathrm{~W}}\right\}$ for the $\mathrm{W}$ case. Indeed, this $\mathrm{W}$ versus MR matching, can be written as follows

$$
\begin{aligned}
a_{\chi}^{+} & =g_{\chi, \mathrm{W}}^{1,+}\left(\Delta \tau_{\mathrm{W}}^{*}\right)^{-\Delta}=\left|\mathbb{Z}_{\chi}^{1,+}\right| \vartheta^{\Delta} \\
\Gamma^{+} & =Z_{\chi, \mathrm{W}}^{+} \chi_{0, \mathrm{~W}}^{+}=\left(\mathbb{Z}_{\chi}^{+}\right)^{-1} \mathbb{X}_{0}^{*,+} \\
B & =Z_{M, \mathrm{~W}} M_{0, \mathrm{~W}}=\mathbb{Z}_{M} \mathbb{M}_{0}^{*}
\end{aligned}
$$

without reduction in the account of the Ising-like universal features estimated in $\mathrm{W}$ (left equations) and MR (right equations).

To obtain the complete description of $\mathrm{W}$, it remains to introduce the intrinsic non-universal parameters involved in the estimation of $\left\{\Delta \tau_{\mathrm{W}}^{*} ; \chi_{0, \mathrm{~W}}^{+} ; M_{0, \mathrm{~W}}\right\}$, as reported in Table 3 (using the notations of the references listed in subline 0 of this Table). Columns 3 to 6 of Table 3 refer to CLM-I \& II, CPM, and MSR, respectively, while the case of TGN is not included due to the numerical forms of the crossover functions.

The non-universal parameter sets involved in each model $\mathrm{W}$ are given in line 1 . In addition, lines 2 to 4 indicate the definitions of the useful sub-parameter combinations introduced in each model. $\Delta \tau_{\mathrm{W}}^{*}$ were given in line 5 while the leading prefactors $\chi_{0, \mathrm{~W}}^{+}$and $M_{0, \mathrm{~W}}$ are reported in lines 6 and 7 . When the $\mathrm{W}$ fits of the experimental data obtained at a finite temperature distance from $T_{c}$ have produced comparable values of the fluid PAD amplitudes $a_{\chi}^{+}$, $\Gamma^{+}$, and $B$ (as in the xenon case of Table 1), Eqs. (47), (48), and (49) mean that $\Delta \tau_{\mathrm{W}}^{*}$, and then, subsequently, $\chi_{0, \mathrm{~W}}^{+}$and $M_{0, \mathrm{~W}}$, are the effectively adjusted quantities, not the individual free parameters of W. Hence, the true non-universal character of the fluid is given as a form of multi-parameter combinations. Therefore, the correlated difficulty is the correct understanding of the nature and the order of magnitude of each non-universal parameter involved in $\left\{\Delta \tau_{\mathrm{W}}^{*} ; \chi_{0, \mathrm{~W}}^{+} ; M_{0, \mathrm{~W}}\right\}$. When the values of the intrinsic non-universal parameters are available in the xenon case, the corresponding numerical values of $\Delta \tau_{\mathrm{W}}^{*}, \chi_{0, \mathrm{~W}}^{+}$, and $M_{0, \mathrm{~W}}$ are given between brackets in lines 5 to 7, columns 5 and 6 of Table 3. However, such multi-parameter combinations can not easily be identified with the similar MR parameters given in column 7 . 
Nevertheless, in the case of $\Delta \tau_{\mathrm{W}}^{*}$, a careful attention focused on the true independent values of this free-parameter combination confirms the existence of a single value for $\Delta \tau_{\mathrm{W}}^{*}$ for CPM (column 5) and MSR (column 6), adjusted above and below $T_{c}$ from the data fitting at finite distance from $T_{c}$. We recall that the added arbitrary condition $\frac{\Lambda}{\left(c_{t}\right)^{\frac{1}{2}}}=\pi$ (see line 8 ) in the CPM case (column 5) leads to a restricted three-free-parameter fitting condition, similar to the one of the MSR case. $\Delta \tau_{\mathrm{W}}^{*}$ values take an Ising-like nature similar with that of $\left(\vartheta_{\mathcal{L}}^{ \pm}\right)^{-1}$ in the MR case since extracted from similar conditions at finite distance from $T_{c}$. We see here why our previous [1] non-analytical scaling estimation of $\left(\vartheta_{\mathcal{L}}^{ \pm}\right)^{-1}$ at a finite (positive and negative) distance away from $T_{c}$ can now be more accurately compared to any crossover theory than heretofore when the values of the free parameter combinations can explicitly and unambiguously be correlated to our local value $\vartheta_{\mathcal{L}}^{ \pm}\left(\Delta \tau^{*}\right)$, subsequently to our induced free parameter $\psi_{\rho}$, and finally to our free leading prefactors $\mathbb{X}_{0, \mathcal{L}}^{*,+}$ and $\mathbb{M}_{0, \mathcal{L}}^{*}$ provided by the use of the MR crossover functions in Ref. [1]. We can in return assume their exact analytic matching, using our MR results of column 7 as references, where the $\operatorname{set}\left\{\vartheta_{\mathcal{L}}^{ \pm} ; \mathbb{X}_{0, \mathcal{L}}^{*,+} ; \mathbb{M}_{0, \mathcal{L}}^{*}\right\}$ obtained in the extended asymptotic domain (here recalled by the subscript $\mathcal{L}$ ), replaces the set $\left\{\vartheta ; g_{0} ; \psi_{\rho}\right\}$ estimated in the preasymptotic domain (see Ref. [1] for details).

\subsection{Similarity of the Non-Universal Parametric Crossover Description Within the PAD}

From the above analyses where all the physical and theoretical PAD descriptions only involve three characteristics quantities, de facto only three theoretical universal ratios $R_{\mathrm{W}}^{1}=\left(\frac{g_{\chi, \mathrm{W}}^{1,+}}{\left|\mathbb{Z}_{\chi}^{1,+}\right|}\right)^{\frac{1}{\Delta}}, R_{\chi}, \mathrm{W}=\frac{\left(\mathbb{Z}_{\chi}^{+}\right)^{-1}}{Z_{\chi, \mathrm{W}}^{+}}$and $R_{M, \mathrm{~W}}=\frac{\mathbb{Z}_{M}}{Z_{M, \mathrm{~W}}}$ are needed to construct the links $\mathrm{W}$ versus MR, through the following equations

$$
\begin{aligned}
\frac{\Delta \tau_{\mathrm{W}}^{*}}{\left(\vartheta_{\mathcal{L}}^{+}\right)^{-1}} & =\left(\frac{g_{\chi, \mathrm{W}}^{1,+}}{\left|\mathbb{Z}_{\chi}^{1,+}\right|}\right)^{\frac{1}{\Delta}}=R_{\mathrm{W}}^{1} \\
\frac{\chi_{0, \mathrm{~W}}^{*,+}}{\mathbb{X}_{0, \mathcal{L}}^{*}} & =\frac{\left(\mathbb{Z}_{\chi}^{+}\right)^{-1}}{Z_{\chi, \mathrm{W}}^{+}}=R_{\chi, \mathrm{W}} \\
\frac{M_{0, \mathrm{~W}}^{*}}{\mathbb{M}_{0, \mathcal{L}}^{*}} & =\frac{\mathbb{Z}_{M}}{Z_{M, \mathrm{~W}}}=R_{M, \mathrm{~W}}
\end{aligned}
$$

The corresponding universal values of Eqs. (50), (51), and (52) are reported in lines 1 to 3 of Table 4. Equation (50) only refer to the confluent correction terms, while leading asymptotic terms are accounted for by Eqs. (51) and (52).

\subsubsection{Matching of the Lowest Confluent Corrections}

We immediately note that our selected $a_{\chi}^{+}$matching using Eq. (50) is similar with the $a_{M}$ matching, such as $\frac{\Delta \tau_{\mathrm{W}}^{*}}{\left(\vartheta_{\mathcal{L}}^{-}\right)^{-1}}=\left(\frac{g_{M, \mathrm{~W}}^{1}}{\mathbb{Z}_{M}^{1}}\right)^{\frac{1}{\Delta}} \equiv R_{\mathrm{W}}^{1}$. The equivalence of the multi-parameter combinations is strictly correct when it is assumed no difference in the universal ratios $\frac{a_{M}}{a_{\chi}^{+}}$ 


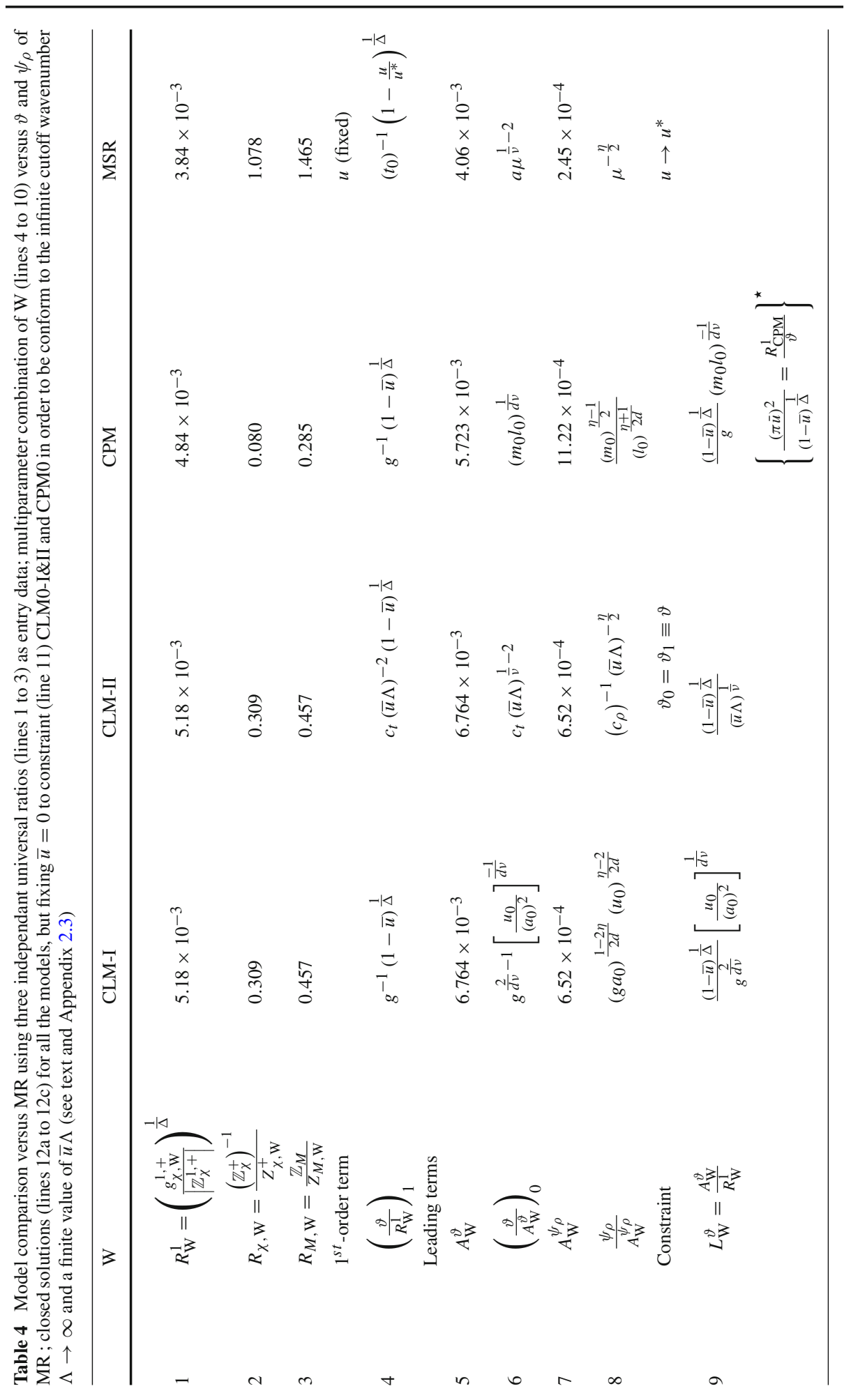




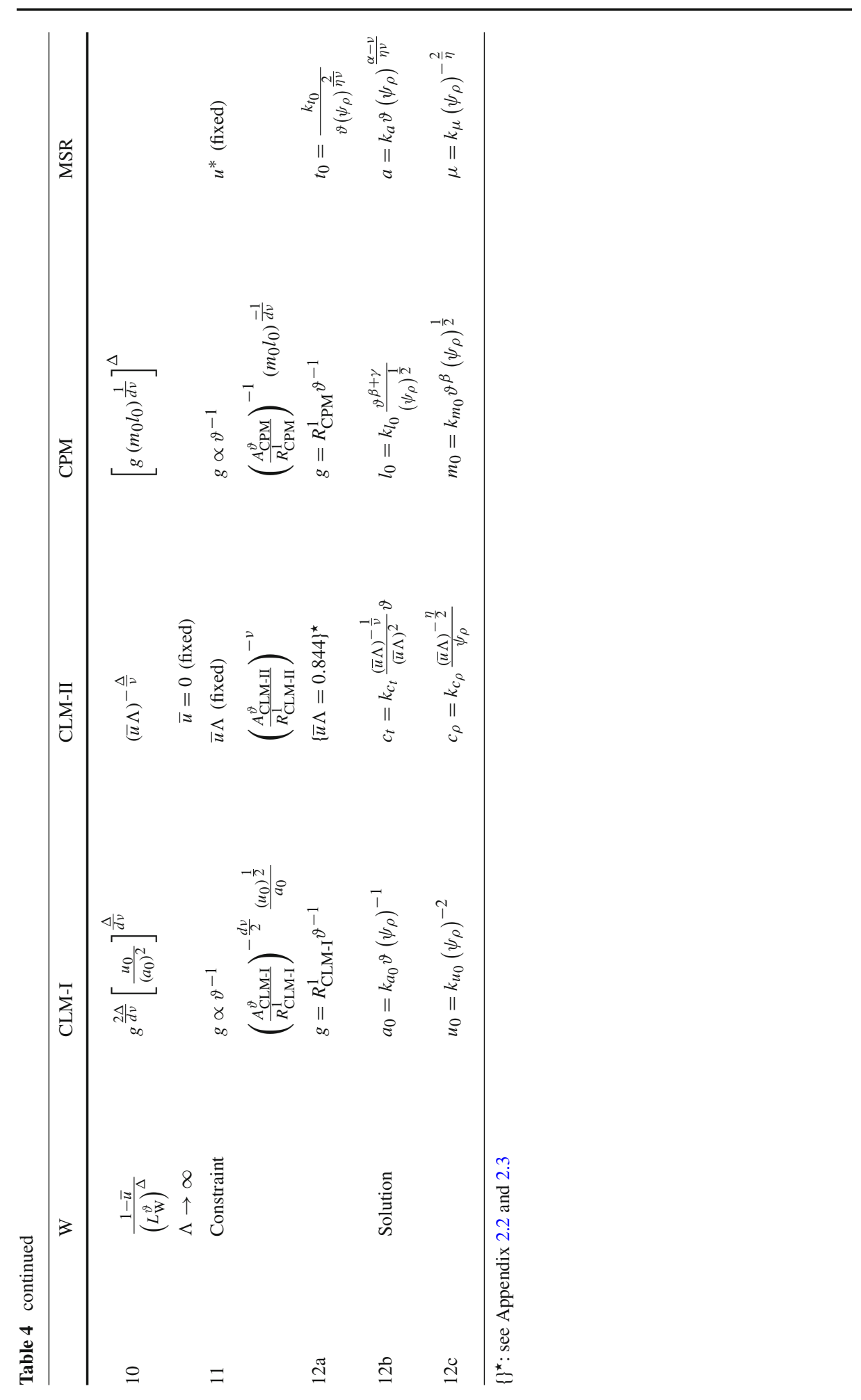


estimated from the different models (see line 13 in Table 2). It remains here essential to note that, in the case of $\mathrm{W}$ as well as in the one of MR, the independent data sources used to extract $\Delta \tau_{\mathrm{W}}^{*}$ and $\vartheta_{\mathcal{L}}^{ \pm}$are based on completely different experiments performed in different finite temperature ranges above or below $T_{c}$. This essential feature is explicit in the MR case where the important distinction using $\left(\vartheta_{\mathcal{L}}^{+}\right)^{-1}$ and $\left(\vartheta_{\mathcal{L}}^{-}\right)^{-1}$ lies in the independent nonanalytic estimations of $\vartheta_{\mathcal{L}}^{+}\left(\Delta \tau^{*}\right)$, where $\vartheta_{\mathcal{L}}^{+} \approx \vartheta$ within a finite, positive temperature range, and $\vartheta_{\mathcal{L}}^{-}$, where $\vartheta_{\mathcal{L}} \approx \vartheta$ within another finite, negative temperature range (see Ref. [1]). Therefore, despite complex forms of the multi-parameter combinations for each $\mathrm{W}$, the following equations (see line 4 of Table 4)

$$
\left(\frac{\vartheta_{\mathcal{L}}^{ \pm}}{R_{\mathrm{W}}^{1}}\right)_{1}=\frac{1}{\Delta \tau_{\mathrm{W}}^{*}}
$$

are the first validation of the Ising-like nature of a single crossover parameter $\Delta \tau_{\mathrm{W}}^{*}$ involved in the first-order confluent matching regardless of W. In Eq. (53), the notation ()$_{1}$ refers to this non-asymptotic construction of the first-order confluent link W versus MR, both values being adjusted at finite distance from $T_{c}$. We note that these $\mathrm{W}$ combinations are then independent of $\mathbb{L}^{\{1 f\}}$. The similar order of magnitude $(\sim 0.23$, see subline 5 of Table 3$)$ between the adjusted values of $\Delta \tau_{\mathrm{W}}^{*}$ is now a direct consequence of the comparable universal values $(\sim 0.6$, see line 1 of Table 2$)$ of $g_{\chi}^{1,+}$, , while the highest value $\left|\mathbb{Z}_{\chi}^{1,+}\right|=8.56347$ explains the highest value of $\frac{1}{\vartheta}=47.225$. We recall that such a confluent matching analysis is not easy for the case of TGN due to the numerical forms of the crossover functions. However, from the comparison between the Ginzburg-number-like $\Delta \tau_{\mathrm{TGN}}^{*} \propto G_{\mathrm{Xe}}^{+}$of Ref. [31] and $\Delta \tau_{\mathrm{MR}}^{*}=\frac{1}{\vartheta}$ of MR, we can reasonably expect that there exist one value $\Delta \tau_{\mathrm{TGN}}^{*} \propto \frac{1}{\vartheta}$, for which the experimental data coincide with the MR curve and the TGN curve over the full temperature range, with the similar level of $0.4 \%$ accuracy (see for example Figure 5 in Ref. [13] for the case of the MR curve). However, the universal value of the prefactor of proportionality is not available since the numerical form of the TGN crossover function can not permit direct access to the corresponding theoretical universal value $g_{\chi, \mathrm{TGN}}^{1,+}$, which is implicitly dependent on the interaction range. Such a situation differs somewhat from the observations of the curve labeled BB in Fig. 3 of Ref. [31], probably due to the arbitrary adjustment of $4.5 \%$ required to bring the additional results of Ref. [46] into line with those of Ref. [14]. Nevertheless, the noticeable difference between the fitting values $G_{\mathrm{Xe}}^{+}=(1.8 \pm 0.2) \times 10^{-2}$ above $T_{c}$ and $G_{\mathrm{Xe}}^{-}=(7 \pm 2) \times 10^{-2}$ below $T_{c}$ still remains not fully understood using only our hypothetized first-order confluent matching for the PAD description.

As the Ising-like nature of the crossover regime is characterized by a single non-universal parameter, the number of adjustable parameters in the models can be reduced by one (a result already noted in the MSR modeling of ${ }^{3} \mathrm{He}$ properties [44]). It remains to see how such a reduction can come about in each model, that is supported by the data reported in lines 5 to 12 of Table 4 and the related following discussion, complemented by the results given in Appendix 2.

\subsubsection{Matching of the Leading Asymptotic Behavior}

From the leading amplitude matching through Eqs. (51) and (52), we can define the following universal quantities $A_{\mathrm{M}}^{\vartheta}$ and $A_{\mathrm{W}}^{\psi_{\rho}}$ 


$$
\begin{aligned}
A_{\mathrm{W}}^{\vartheta} & =\left[\frac{R_{\chi}, \mathrm{W}}{\left(R_{M, \mathrm{~W}}\right)^{2}}\right]^{\frac{1}{d v}}\left(\mathbb{L}^{\{1 f\}}\right)^{-\frac{1}{v}} \\
A_{\mathrm{W}}^{\psi_{\rho}} & =\left[\left(R_{\chi}, \mathrm{W}\right)^{\beta}\left(R_{M, \mathrm{~W}}\right)^{\gamma}\right]^{\frac{-1}{d v}}\left(\mathbb{L}^{\{1 f\}}\right)^{-\frac{\gamma+\beta}{v}}
\end{aligned}
$$

$A_{\mathrm{W}}^{\vartheta}$ and $A_{\mathrm{W}}^{\psi_{\rho}}$, which replace $R_{\chi}, \mathrm{W}$ and $R_{M, \mathrm{~W}}$, are theoretical universal quantities reported in lines 5 and 7 of Table 4, which not only depend on combinations of non-analytical powers of $R_{\chi, \mathrm{W}}^{+}$and $R_{M}$,W defined by Eqs. (51) and (52), but also on explicit non-analytic powers of $\mathbb{L}^{\{1 f\}}$. Hence, our separated validation of the identity $\vartheta^{ \pm} \equiv \vartheta$ above and below $T_{c}$ made in Ref. [1], combined with the explicit contribution of $\mathbb{L}^{\{\tilde{f}\}}$ in Eqs. (54) and (55), permit the following independent equations to be distinguished, thanks to the universal aymptotic scaling

$$
\begin{aligned}
\left(\frac{\vartheta}{A_{\mathrm{W}}^{\vartheta}}\right)_{0} & =J_{\mathrm{W}} \\
\frac{\psi_{\rho}}{A_{\mathrm{W}}^{\psi_{\rho}}} & =K_{\mathrm{W}}
\end{aligned}
$$

$J_{\mathrm{W}}$ and $K_{\mathrm{W}}$ are the corresponding non-universal multi-parameter combinations given in lines 6 and 8. Equations (56) and (57) introduce additional physical meanings from the $\vartheta$ versus $J_{\mathrm{W}}$ and $\psi_{\rho}$ versus $K_{\mathrm{W}}$ comparisons, respectively. The notation ()$_{0}$ refers to this asymptotic construction of the link $\vartheta$ versus $J_{\mathrm{W}}$. A single common expression for $\left(\vartheta_{\mathcal{L}}^{ \pm}\right)_{1}$ from Eq. (53) and $\left(\vartheta_{\mathcal{L}}^{ \pm}\right)_{0}$ from Eq. (56) corresponds to an unambiguous link $\vartheta$ versus $\Delta \tau_{\mathrm{W}}^{*}$ observed at finite distances from $T_{c}$ (see also Appendix 2.2 for the specific case of four adjustable parameters in CLM-I\&II and CPM). As a consequence, the MR equations $\frac{\chi_{T}^{*}}{\chi_{r}^{+}}=\chi_{\text {th }}\left(\vartheta \Delta \tau^{*}\right)$ and $\frac{\Delta \tilde{\rho}_{L V}}{M_{r}}=m_{\text {th }}\left(\vartheta\left|\Delta \tau^{*}\right|\right)$ [see corresponding Eq. (5)] infer the similar W equations $\frac{\chi_{T}^{*}}{\chi_{n, \mathrm{~W}}^{+}}=F_{\chi}, \mathrm{W}\left(\frac{\Delta \tau^{*}}{\Delta \tau_{\mathrm{W}}^{*}}\right)$ and $\frac{\Delta \tilde{\rho}_{L V}}{M_{n, \mathrm{~W}}}=F_{M, \mathrm{~W}}\left(\frac{\Delta \tau^{*}}{\Delta \tau_{\mathrm{M}}^{*}}\right)$, where the functions $F_{\chi, \mathrm{W}}\left(\frac{\Delta \tau^{*}}{\Delta \tau_{\mathrm{W}}^{*}}\right)$ and $F_{M, \mathrm{~W}}\left(\frac{\Delta \tau^{*}}{\Delta \tau_{\mathrm{W}}^{*}}\right)$ are expected to be universal. Practically, $\left\{\chi_{n, \mathrm{~W}}^{+} ; M_{n, \mathrm{~W}}\right\}$ are the readily asymptotic independent quantities extracted from the data fittings with a single crossover parameter above and below $T_{c}$. Correlatively, we note that the normalization of the susceptibility and coexisting density data by their corresponding leading power law terms, i.e., $\frac{\kappa_{T}^{*}}{\Gamma^{+}\left(\Delta \tau^{*}\right)^{-\gamma}}$ and $\frac{\Delta \tilde{\rho}_{L V}}{B\left|\Delta \tau^{*}\right|^{\beta}}$, are thus not fully convenient to compare the shape of the crossover functions in simple fluids.

Indeed, such similar universal forms of the critical-to-classical crossover functions were already calculated in Refs. [40] and [41], introducing the so-called effective Ginzburg number, noted $\mathrm{Gi}_{P}$. The condition $\frac{\Delta \tau^{*}}{\mathrm{Gi} P} \gg 1$ is thus a practical criterion to define the limit of validity for the mean-field regime of the singular behavior of the property $P^{*}\left(\Delta \tau^{*}\right)$, starting from the classical (three-term) Landau expansions of the free energy close to the Gaussian fixed point. Hence, the renormalization of the non-universal mean-field parameters leads to the universal functions $\chi_{T}^{*} \mathrm{Gi}_{\chi} \bar{\chi}_{0, \mathrm{Gi}_{\chi}}=F_{\mathrm{Gi}_{\chi}, \chi, \mathrm{W}}\left(\frac{\Delta \tau^{*}}{\mathrm{Gi}_{\chi}}\right)$ and $\Delta \tilde{\rho}_{L V} \mathrm{Gi}_{M} \bar{M}_{0, \mathrm{Gi}_{M}}=F_{\mathrm{Gi}_{M}, M, \mathrm{~W}}\left(\frac{\Delta \tau^{*}}{\mathrm{Gi}_{M}}\right)$ where $\bar{\chi}_{0, \mathrm{Gi}_{\chi}}$ and $\bar{M}_{0, \mathrm{Gi}_{M}}$ are non-universal (mean-field-like) parameters. The added subcripts $\mathrm{Gi}_{\chi}$ and $\mathrm{Gi}_{M}$ recall for the corresponding construction of the Ising-like limit of these universal crossover functions. However, the derivations of such universal forms are strictly valid only in the infinite cutoff approximation $(\Lambda \rightarrow \infty)$, as in the MSR scheme where $t_{0}=\frac{\mu^{2}}{a}$ appears well like an effective Ginzburg number. Finally, a closed comparison $\mathrm{W}$ 
versus MR can only be performed for a W model with three non-universal parameters, such as MSR (see also Appendix). Similarly, when is added the infinite cutoff approximation ( $\Lambda=\infty, \bar{u}=0$, with $\bar{u} \Lambda$ finite) in the $\mathrm{W}_{+}$cases, the functional forms of $\Delta \tau_{\mathrm{W}}^{*}, \chi_{0, \mathrm{~W}}^{+}$, and $M_{0, \mathrm{~W}}$ (see lines 5 to 7 of Table 3 ) show that the subparameter $g$ of CLM0-I and CPM0, or the explicit ratio $\frac{c_{t}}{(\bar{u} \Lambda)^{2}}$ of CLM0-II, appear well as the effective Ginzburg-like numbers.

In the general four parameter cases with $\bar{u} \neq 0$, the situation, however, appears more complex when starting from the functional forms of $\Delta \tau_{\mathrm{CLM}-\mathrm{I}}^{*} \equiv \Delta \tau_{\mathrm{CPM}}^{*}=g(1-\bar{u})^{-\frac{1}{\Delta}}$ and $\Delta \tau_{\mathrm{CLM}-\mathrm{II}}^{*}=\left(c_{t}\right)^{-1}(\bar{u} \Lambda)^{2}(1-\bar{u})^{-\frac{1}{\Delta}}$. Thus calculating

$$
\begin{aligned}
\chi_{n, \mathrm{CLM}-\mathrm{I}}^{+} & =\left(a_{0}\right)^{-1} g^{-1}(1-\bar{u})^{\frac{\gamma}{\Delta}} \\
\chi_{n, \mathrm{CLM}-\mathrm{II}}^{+} & =\left(c_{\rho}\right)^{-2}(\bar{u} \Lambda)^{-2}(1-\bar{u})^{\frac{\gamma}{\Delta}} \\
\chi_{n, \mathrm{CPM}}^{+} & =\frac{m_{0}}{l_{0}} g^{-\gamma}(1-\bar{u})^{\frac{\gamma}{\Delta}} \\
M_{n, \mathrm{CLM}-\mathrm{I}} & =g^{\frac{1}{2}}\left(\frac{a_{0}}{u_{0}}\right)^{\frac{1}{2}}(1-\bar{u})^{-\frac{\beta}{\Delta}} \\
M_{n, \mathrm{CLM}-\mathrm{II}} & =\left(c_{\rho}\right)^{-1}(\bar{u} \Lambda)^{\frac{1}{2}}(1-\bar{u})^{-\frac{\beta}{\Delta}} \\
M_{n, \mathrm{CPM}} & =m_{0} g^{\beta}(1-\bar{u})^{-\frac{\beta}{\Delta}}
\end{aligned}
$$

we observe that the powered $(1-\bar{u})$ term remains explicit in each asymptotic combination (see also the discussion in Appendix 2.2). Obviously, the fact that the two crossover parameters $g ; \bar{u}$, or $\bar{u} \Lambda ; \bar{u}$, explicitely appear in leading normalized amplitudes seems not necessary in a three-parameter description of a simple fluid. That opens the route to a possible introduction of any arbitrary condition that reduces by one the number of free nonuniversal parameters in these $\mathrm{W}_{+}$cases.

Nevertheless, here occurs the main modeling differences with the MR case, which lie in the fact that $\left(\vartheta_{\mathcal{L}}^{ \pm}\right)^{-1}$ is only Ising-like-asymptotic in nature and unique for all the singular behaviors of the fluid properties along the critical isochore. In addition, $\chi_{r}^{+}=\left(\psi_{\rho}\right)^{2}\left(\mathbb{L}^{\{1 f\}}\right)^{d}$ and $M_{r}=\psi_{\rho}\left(\mathbb{L}^{\{1 f\}}\right)^{d}$ are thus readily, non-classical, metric-like quantities in the MR case, i.e., not dependent on $\vartheta$ and only dependent on $\psi_{\rho}$ and $\mathbb{L}^{\{1 f\}}$ (due to the extensive nature of the selected properties). When we assume a universal value for the non-dimensional master length $\mathbb{L}^{\{1 f\}}$ in order to maintain a single length unit for dimensionless correlation and thermodynamic properties, $\psi_{\rho}$ is thus the second MR scale factor which characterizes all the asymptotic singular behaviors of the fluid properties along the critical isotherm. Hence, for $\mathrm{W}_{+}$cases with four non-universal parameters, the remaining problem is to perform a similar selective analysis for the multiparameter combinations $\left\{\chi_{0, \mathrm{~W}}^{+}, M_{0, \mathrm{~W}}\right\}$ versus $\left\{\mathbb{X}_{0, \mathcal{L}}^{*,+}, \mathbb{M}_{0, \mathcal{L}}^{*}\right\}$, or $\left\{\chi_{n, \mathrm{~W}}^{+}, M_{n, \mathrm{~W}}\right\}$ versus $\left\{\chi_{r}^{+}, M_{r}\right\}$. This analysis is given in Appendix 2 where the expected unequivocal relations between the respectively non-universal parameter sets $\left\{g ; a_{0} ; u_{0}\right\}-\left\{\bar{u} \Lambda ; c_{t} ; c_{\rho}\right\}-\left\{g ; m_{0} ; l_{0}\right\}$-versus $\left\{\vartheta ; \psi_{\rho} ; g_{0}\right\}$ are provided for CLM0-I\&II and CPM0 cases with infinite limit of the cutoff wave number. Their validity remains obviously restricted to a simple fluid belonging to the universality subclass of the one-component fluid systems, i.e., such as $g_{0} \alpha_{c}=\mathbb{L}^{\{1 f\}}$ [see Eq. (16)], as demonstrated through the bypass using their comparison to the three-parameter set $\{u ; a ; \mu\}$ in MSR case. Finally, the crossover modelling uniqueness is well demonstrated through the solutions reported in lines 11 and $12 \mathrm{a}-\mathrm{c}$ of Table 4 based on the detailed analysis of Appendix 2. The non-universal nature 
of the simple fluid appears well characterized by introducing only two scale factors, whose values can be estimated when their explicit links to $Y_{c}$ and $Z_{c}$ are formulated (as in the case of CMM [13]).

\section{Conclusion}

The xenon crossover modeling in Ref. [1] has demonstrated the primordial interest of the accurate MR mean crossover functions by determining precise values of the non-universal scale factors which characterize its critical scaling behavior beyond the PAD. In this work, we demonstrate the similarity of the results analyzed by the different crossover models. Potential applications of any three parameter crossover model are now extended for the case of equation-of-state models. It also becomes essential that the development of future theoretical crossover functions shows explicit closed forms accounting for resummation of infinite Wegner-like expansions along the critical paths (especially for the still lacking theoretical functions along the critical isotherm). By construction, these functions should also have an Ising-like, well-defined asymptotic limit with fully explicit integration of the most precise estimates of the universal values for the critical exponents and the amplitude combinations (including the first-order of the confluent corrections to scaling).

Up until the present day, the fundamental interest for developing a universal scaled closed form for the equation-of-state of simple fluids is concomitant to the quest of the true asymptotic singular behavior, which always remains as conundrum to the experimentalists performing studies closer and closer to the vapor-liquid critical point [47]. Nevertheless, in the absence of numerous accurate measurements within the PAD, the actual level of knowledge of the universal features of simple fluids seems compatible with only three adjustable free parameters in any crossover model of the equation-of-state. In such a case that accurately accounts for the first-order confluent corrections-to-scaling, the analyses of the singular properties measured at finite distance from $T_{c}$, is important to check that the expected reduction by one of the free parameters occurs well beyond their respective PAD. This very precise estimation of the single temperature scaling factor along the critical isochore is essential to sustain the theoretical construction of the critical-to-classical crossover along the renormalized trajectory, where only one family of the corrections-to-scaling terms (governed by the specific lowest value of the exponent $\omega$, or $\Delta=\omega \nu$, equivalently) are resummed into the crossover functions.

In addition, until now, the introduction of the (property-dependent) Ginzburg number to rescale the mean-field temperature appeared as a convenient tool to define an order of magnitude for the limit of the critical mean-field crossover behavior of each property. Such a limiting criterion starts from the Gaussian fixed point of the renormalized trajectory and needs the introduction of (a minima) three unknown, mean-field-like, non-universal parameters in the classical Hamiltonian (including thus a square-gradient term). However, a noticeable difference must now be underlined in such an asymptotic crossover within the critical domain with our introduction of the Ising-like temperature scaling factor $\vartheta^{-1}$, which starts from a minute asymptotical distance to the physical critical point. In the latter situation, the single (i.e., property-independent), fluid-dependent value of $\vartheta$ can only be used over the so-called extended asymptotic domain. $\vartheta^{-1}$ defines the true validity range of the Ising-like critical crossover estimated along the renormalized trajectory close to the non-trivial fixed point. In return, it was thus generally assumed that the true initial Hamiltonian points of these actual fluids with comparable short-ranged molecular interaction lie very close to the renormal- 
ized trajectory. Indeed, a physical reference to a completed theoretical critical-to-classical crossover from the Gaussian fixed point to the non-trivial fixed point, was, to our knowledge, never observed in the subclass of simple fluids. Such a situation is easily understood as the mean field approximation takes a plausible physical meaning only close to the triple point of such simple fluids. More generally, the mean-field-like van der Waals equation-of-state, or any cubic equation-of-state only remains a simple convenient concept to account for attractive molecular forces in a phenomenlogical manner, but always incorrect on the basis of fundamental approaches, for example, developping extended virial forms for the dense gas properties or specific liquid theories.

Obviously, any sophisticated crossover model of the equation-of-state incorporating more than three free parameters is out of the scope of this paper, where the main central interest concerns the physical description of the asymptotic critical crossover in simple fluids with shortranged molecular interaction. For instance, a fourth non-universal parameter can be related to the contributions of one supplementary irrelevant field. In such a case, the account for the effects of another confluent exponent in the Wegner expansions seems needed for theoretical coherence. Its can also be related to a more complex physical understanding of the microscopic nature introduced in the model, such for example an additional reference to a mesoscopic length or a molecular modification of the range of interaction. The difficulty appears generally in the precise experimental characterization of the expected PAD behavior in the related complex physical systems where the order parameter choice remains not unequivocal, especially introducing addition mixing of the field variables. Such a more complicated modeling strategy differs significantly from the one given initially in Ref. [18], which was mainly focused on the controlled reduction of the number of adjustable free parameters to support the experimentalists in their analyses of the measured data in simple fluids closer and closer to their gas-liquid critical point. Unfortunately, since the 1980s up until the present day, no theoretical progress has been able to account correctly for the true physical crossover behavior in simple fluids, observed beyond the extended critical asymptotic domain when $\xi_{\operatorname{expt}}\left(\left|\Delta \tau^{*}\right|\right) \simeq$ $\alpha_{c}$, as illustrated by the crossover behavior of the effective exponents in Fig. 1 of Ref. [18] for the susceptibility case and Fig. 3 of Ref. [43] for the order parameter density case.

From this Ising-like standard situation provided by critical simple fluids, the real extension and amplitude of the singular behavior of the fluid properties can be estimated in a similar manner for any one-component fluid for which the vapor-liquid critical point is localized in the $p V T$ phase surface by use of the master crossover functions given in [9] and/or the CMM model of the equation-of-state given in [13]. Therefore, the comparisons between simple fluid experiments and theory will be improved as they can be performed, now [47] and in the future, without adjustable non-universal parameter.

Acknowledgments Part of the work (CL, SM, YG) was supported by CNES. Part of the work (IH) was carried out at Jet Propulsion Laboratory, California Institute of Technology, under a contract with NASA.

\section{Appendix 1: Brief Status of the Crossover Modelling of the Xenon Properties}

\section{Appendix 1.1: Fitting Models and Xenon Data Sources Used in Table 1}

All the PAD amplitudes given in Table 1 have been extracted from the fits of the experimental data obtained beyond the xenon PAD, still fixing the exponent values of $\gamma, \beta$, and $\Delta$ in the theoretical crossover functions calculated by the different theoretical models $\mathrm{W}$ referenced in 
Sect. 3.1. $\Gamma^{+}$is non-available in [26] due to the fit of the effective exponent $\gamma_{e}\left(\Delta \tau^{*}\right)$ inferred by the susceptibility data, while $a_{\chi}^{+}$and $a_{M}$ are non-available in [31] due to the numerical form of the calculated crossover functions. However, all these models are consistent with the renormalization group theory since their Ising-like universal features within the PAD are very similar, introducing, a minima, three non-universal parameters to characterize each physical system belonging to the $O$ (1) universality class (see Table 2).

More precisely, the main experimental data sources involved in such fittings were obtained in the 1980s. They are the susceptibility data and the turbidity data of Güttinger and Cannell [14], the vapor-liquid coexisting density data measured by Närger and Balzarini [15] in two different samples of xenon, and the specific heat data at constant volume of Edwards et al. [48]. The temperature ranges are typically within $10^{-4} \lesssim\left|\Delta \tau^{*}\right| \lesssim 10^{-1}$. Accordingly, from 84's up to now, we have been interested in the following modelings: (i) The MR6 max testfunctions calculated in Ref. [16] were used in Ref. [18] to fit susceptibility, turbidity and specific heat data above $T_{c}$; (ii) The CLM crossover function for susceptibility, as detailed for example in Ref. [45], was used in Ref. [26] to fit the normalized susceptibility data above $T_{c}$; (iii) The CPM crossover functions calculated in Ref. [28] were used in Ref. [13] to fit the susceptibility, turbidity, correlation length data above $T_{c}$ and the coexisting density data below $T_{c}$. We note that, in this same Ref. [13], the CMM crossover functions were also compared to CPM and MR ones from the joint fit of the susceptibility and coexisting density data for $T \lessgtr T_{c}$; (iv) The MSR crossover functions calculated in Ref. [22] were used in Ref. [21] to fit the susceptibility data above $T_{c}$; (v) The TGN numerical functions calculated in Ref. [29] were used in Ref. [31] to separately fit the susceptibility data above $T_{c}$ and coexisting density data below $T_{c}$.

We also note that the two-phase domain below $T_{c}$ have been analyzed only from the Närger and Balzarini [15] measurements at finite distance from $T_{c}$. In the following, three additional remarks concerning the fitting results obtained at finite temperature distance above $T_{c}$ and a final comparative comment concerning the fitting results including experimental results obtained within the (one-phase and two-phase) xenon PAD can be formulated.

\section{Appendix 1.2: Fitting Analyses of the Normalized Susceptibility Above $T_{c}$}

The important preliminary remark is that the normalized presentation of the susceptibility data in Ref. [14], avoids de facto the determination of the value of the physical amplitude $\Gamma^{+}$in fitting analyses where the critical temperature was also considered as an adjustable parameter. That provides an opportunity for first analyzing the normalized susceptibility data-or the effective exponent $\gamma_{e}\left(\Delta \tau^{*}\right)$, equivalently-with only a single adjustable parameter in the crossover function where $\Delta$ takes the fixed theoretical value. The clear understanding of such a single parameter fitting can be found in Fig. 1 of Ref. [18] and in Fig. 2 (Xe) of Ref. [26]. Unfortunately, four decades latter, the true crossover behavior to the mean-field value of $\gamma_{e}\left(\Delta \tau^{*}\right)$ indicated in Fig. 1 of Ref. [18], always remains unexpected by any crossover theory (with a similar remark for $\beta_{\text {eff }}\left(\Delta \tau^{*}\right)$ in Fig. 3 of Ref. [43]).

\section{Appendix 1.3: Fitting Analyses of the Normalized and Dimensional Susceptibility Above $T_{c}$}

Fitting of the dimensional susceptibility data shows that $\Gamma^{+}$value appears mainly dependent on the theoretical value of the fixed exponent $\gamma$ and the selected value of the reference susceptibility data. Therefore, all the fitting results given in lines 1 to 5 of Table 1 have similar behaviors in the interpolated-PAD in the sense where the level of $\sim 0.4 \%$ accuracy 
was always revealed in the experimental range $10^{-4} \lesssim \Delta \tau^{*} \lesssim 5 \times 10^{-2}$ (with no significative variation of $T_{c}$ observed) using the (single parameter) rescaled temperature fields in each model. Consequently, such a similarity implies that the single non-universal values of $\vartheta_{\mathcal{L}}^{+}$ or $\Delta \tau_{\mathrm{W}}^{*}$, adjusted far away from $T_{c}$, are directly correlated to the true, but unknown, nonuniversal value of $a_{\chi}^{+}$, the latter amplitude being prominently close to $T_{c}$ in characterizing the Ising-like nature of xenon. Despite such expected links between the adjusted $\Delta \tau_{\mathrm{W}}^{*}$ values and the true $a_{\chi}^{+}$value, the typical uncertainty of this $a_{\chi}^{+}$value can be estimated at $\sim 50 \%$ from Table 1 results. In addition, the true uncertainty of the $\Gamma^{+}$value is certainly in the range of a few $\%$, a level comparable to the dispersion of the $\Gamma^{+}$values reported in the Güttinger and Cannell's fitting results of Ref. [14] (assuming $1 \%$ experimental accuracy on $\kappa_{T \text {,expt }}^{*}$ data) and confirmed by the subsequent analyses reported in Table 1 (fixing the exponent values). Such an uncertainty level on $\Gamma^{+}$is too large to obtain an indirect accurate determination of $a_{\chi}^{+}$(or $\vartheta$, equivalently) within the PAD. As an immediate consequence, all the data fittings at finite distance from $T_{c}$ with more than one single adjustable free parameter (as in the fitting cases of lines 1 to 5 in Table 1) are not able to extract the single asymptotic scaling factor $\Delta \tau_{\mathrm{W}}^{*}$.

\section{Appendix 1.4: Fitting Analyses of Other Data Sources Above $T_{c}$}

From the initial results of Ref. [18] using the MR6 crossover functions with $\vartheta_{\mathcal{L}}^{+}$free, we have shown here the limited interest for supplementary fitting of xenon singular properties (in a finite temperature range above $T_{c}$, along the critical isochore) using CLM, CPM, MSR or TGN to get an accurate estimation of their intrinsic nonuniversal parameters. For instance, in Ref. [18], the fitting of the GC turbidity data above $T_{c}$ where $\vartheta_{\mathcal{L}}^{+}, \Gamma^{+}$and $\xi_{0}^{+}$are left free, involved as a result $\vartheta_{\mathcal{L}}^{+}=0.02 \pm 0.03$, i.e., a noticeably poor estimation of the scale factor $\vartheta_{\mathcal{L}}^{+}$. Therefore, here we can stress that the Ising-like PAD description of $\kappa_{T}$ and $\xi$ can only be retrieved when are fixed the values of $\vartheta_{\mathcal{L}}^{+}=0.0191$ in the MR6 1984's fitting and $\vartheta=0.0211752$ in the recent MR7 re-analysis [13] without adjustable parameter.

Similarly, the fitting results of the specific heat data [48] in the temperature range $10^{-4} \lesssim$ $\Delta \tau^{*} \lesssim 10^{-3}$ were also reported in Ref. [18]. Unfortunately, it has not been possible to obtain a good determination of the scale factor $\vartheta_{\mathcal{L}}^{+}$as these specific heat measurements are not as accurate as those of the scattered light intensity and turbidity. The fit fixing $\vartheta_{\mathcal{L}}^{+}=0.0191$ with free leading amplitude $A^{+}$and (here not important) free background constant $B_{\text {exp }}$, was without systematic deviation within a supposed experimental error of $2 \%$. The resulting adjusted value of $A^{+}$was compatible with $\xi_{0}^{+}=0.186 \pm 0.001 \mathrm{~nm}$ by using the universal amplitude combination $R_{\xi}^{+}=\xi^{+}\left(A^{+}\right)^{\frac{1}{d}}=0.27$. Obviously, similar results are expected using CLM, CPM, MSR or TGN with similar universal value for $R_{\xi}^{+}$(see line 11 of Table 2).

\section{Appendix 1.5: Fitting Analyses Including Experimental Data Within the Xenon PAD}

A small number of theoretical analyses have addressed the xenon singular description from experimental data obtained very close to its vapor-liquid critical point, i.e., within the xenon PAD. To our knowledge, only the observations of the Fraunhofer interference patterns due to the Earth's-gravity-induced density profiles performed in the $1980 \mathrm{~s}$ very close to $T_{c}$ have provided the indirect access to the susceptibility data and the coexisting density data within the PAD, through the measurements of the optical phase quantity $\tilde{\rho}-\kappa_{T}^{*} \tilde{\mu}$ and the fringe angle [49-52]. However, the analyses of these interference patterns were affected by the complexity of the expressions for $\tilde{\rho}-\kappa_{T}^{*} \tilde{\mu}$, which were, at the date, highly dependent on the selected parametric models of the scaled equation-of-state. Today, it is well-recognized 
that such parametric models are never in full conformity with the universal features calculated from theoretical renormalization methods [53]. In addition, only two singular properties were not intrinsically able to validate the Ising-like crossover nature of xenon characterized by three non-universal parameters. Nevertheless, it remains interesting to note that some fitting analyses have been performed by using the Wilcox-Estler scaled parametric equation of state [54] (see also detail in Ref. [50]) with only two free exponents ( $\beta$ and $\gamma$ ), i.e., in conformity with the two-scale factor universality. These analyses, only considering the asymptotic contribution of the leading power law terms $\Delta \tilde{\rho}_{L V \text {,expt }}=B\left|\Delta \tau^{*}\right|^{\beta}$ and $\kappa_{T, \text { expt }}^{*}=\Gamma^{+}\left(\Delta \tau^{*}\right)^{-\gamma}$, have thus provided access to the exponent-amplitude pairs $\{\beta ; B\}$ and $\left\{\gamma ; \Gamma^{+}\right\}$for the free and fixed values of the exponents. The corresponding results were $\{\beta=0.329 \pm 0.005 ; B=1.48 \pm 0.06\},\left\{\gamma=1.23 \pm 0.003 ; \Gamma^{+}=0.062 \pm 0.006\right\}$ with free exponents [51] and $\{\beta=0.325 ; B=1.42 \pm 0.03\},\left\{\gamma=1.24 ; \Gamma^{+}=0.058 \pm\right.$ $0.002\}$ with fixed exponents [52] in agreement with the results reported in Table 1. This good level of agreement was initially underlined by Güttinger and Cannell for the case of their determination of the interpolated $\left\{\gamma ; \Gamma^{+}\right\}$pair because the two experiments rely on completely different effects and there is no region of overlap between the temperature distances from $T_{c}$ of the two data sets.

\section{Appendix 2: Two-Scale Factor Similarity of the Crossover Models with $\Lambda \rightarrow \infty$}

\section{Appendix 2.1: MSR Versus MR}

Using the notations and the results of Ref. [22], it is immediate to see that the MSR nonuniversal quantities needed to construct the universal crossover functions $F_{\chi, n, \operatorname{MSR}}\left(\frac{\Delta \tau^{*}}{t_{0}}\right)$ and $F_{M, n, \mathrm{MSR}}\left(\frac{\Delta \tau^{*}}{t_{0}}\right)$ are $\chi_{n, \mathrm{MSR}} \equiv \chi_{0} \propto \mu^{-2}, M_{n, \mathrm{MSR}} \equiv \phi_{0} \propto \mu^{\frac{1}{2}}$, and $\Delta \tau_{\mathrm{MSR}}^{*} \equiv t_{0}=\frac{\mu^{2}}{a}$. De facto, only $a$ (the renormalized temperature-like scaling parameter) and $\mu$ (the arbitrary reference length needed in the estimation of the renormalized coupling parameter $u$ ) are involved in such a construction (see Sect. 5.1). Therefore, the implicit role of $u$ remains subtle to understand as the temperature scaling factor $t_{0}=\frac{\mu^{2}}{a}$ is only defined for the fixed point limit $u=u^{*}$, i.e., without any reference to the mean-field regime. That requires checking the goodness of the fit versus $\left(1-\frac{u}{u^{*}}\right)$ in order to control the expected power-law dependence of $\mu \propto\left(1-\frac{u}{u^{*}}\right)^{\frac{v}{\Delta}}$ and $a \propto\left(1-\frac{u}{u^{*}}\right)^{\frac{2 v-1}{\Delta}}$, and the resulting power-law dependence of $t_{0}=\frac{\mu^{2}}{a} \propto\left(1-\frac{u}{u^{*}}\right)^{\frac{1}{\Delta}}$ involved in the expressions of the first Wegner amplitudes where highorder terms were ignored. The main consequence is that only two out of the three- $\{u ; \mu ; a\}$ non-universal parameters are relevant fitting parameters. As expected, the use of a free ratio $\frac{u}{u^{*}}$ to be adjusted in the fit leaves the fit goodness unchanged for $\left(1-\frac{u}{u^{*}}\right) \leq 10^{-3}$, while the adjusted $\mu$ and $a$ remain the two non-universal parameters characteristic of the physical fluid. The small value of $\left(1-\frac{u}{u^{*}}\right)$ explains the expected large difference between the magnitude of $a$ and $\mu$. For example $u=0.999 u^{*}, a=0.333 \pm 0.012$ and $\mu=(2.97 \pm 0.33) \times 10^{-4}$ in the xenon case reported in Ref. [21], while, from the data fitting, the adjusted magnitudes of the asymptotic multiparameter combinations are on a single decade range (see sublines 6 and 7, column 6 of Table 3) and comparable to the corresponding calculated ones in the case of MR (see column 7). Therefore, considering column 5 of Table 4 with a fixed $u$ value 
and using Eqs. (51) and (52), the two formal parameter links MSR versus MR are as follows (lines $12 \mathrm{~b}$ and $12 \mathrm{c}$ ),

$$
\begin{aligned}
& a=k_{a} \vartheta\left(\psi_{\rho}\right)^{\frac{\alpha-v}{\eta v}} \\
& \mu=k_{\mu}\left(\psi_{\rho}\right)^{-\frac{2}{\eta}}
\end{aligned}
$$

with $t_{0}=\frac{\mu^{2}}{a}=k_{t_{0}} \vartheta^{-1}\left(\psi_{\rho}\right)^{\frac{-2}{\eta v}}$ (line 12a). Obviously, $\vartheta=A_{\mathrm{MSR}}^{\vartheta}\left(t_{0}\right)^{-1} \mu^{\frac{1}{v}}$ and $\psi_{\rho}=$ $A_{\mathrm{MSR}}^{\psi_{\rho}} \mu^{-\frac{\eta}{2}}$, where the values (lines 5 and 7) of $A_{\mathrm{MSR}}^{\vartheta}$ and $A_{\mathrm{MSR}}^{\psi_{\rho}}$ are calculated from Eqs. (54) and (55). Hence, the prefactors in Eqs. (64) and (65) are

$$
\begin{aligned}
& k_{a}=A_{\mathrm{MSR}}^{\vartheta}\left(A_{\mathrm{MSR}}^{\psi_{\rho}}\right)^{\frac{v-\alpha}{\eta v}} \\
& k_{\mu}=\left(A_{\mathrm{MSR}}^{\psi_{\rho}}\right)^{\frac{2}{\eta}}
\end{aligned}
$$

with $k_{t_{0}}=\frac{\left(k_{\mu}\right)^{2}}{k_{a}}=\frac{\left(A_{\mathrm{MSR}}^{\psi_{\rho}}\right)^{\frac{2}{\eta v}}}{A_{\mathrm{MSR}}^{\vartheta}}$. The MSR versus MR comparison is closed. In addition the dimensional length scaling factor $l_{0}$ (see last line of Table 2) in MSR is identical to the length unit $\alpha_{c}$ of Eq. (22) in MR. Consequently, introducing the universal ratios $R_{C, \mathrm{~W}}^{+}=\frac{\left(\mathbb{Z}_{C}^{+}\right)^{-1}}{Z_{C, \mathrm{~W}}^{+}}$or $R_{\xi, \mathrm{W}}^{+}=\frac{\left(\mathbb{Z}_{\xi}^{+}\right)^{-1}}{Z_{\xi, \mathrm{W}}^{+}}$, such as $1 \equiv R_{C, \mathrm{~W}}^{+} \frac{R_{\chi, \mathrm{W}}}{\left(R_{M, \mathrm{~W}}\right)^{2}}, 1 \equiv R_{C, \mathrm{~W}}^{+}\left(R_{\xi, \mathrm{W}}^{+}\right)^{d}$ or $1 \equiv\left(R_{\chi, \mathrm{W}}^{+}\right)^{-d} \frac{R_{\chi, \mathrm{W}}}{\left(R_{M, \mathrm{~W}}\right)^{2}}$ from our hypothesis on identical universal features whatever $\mathrm{W}$, and noting thus that $A_{\mathrm{MSR}}^{\vartheta} \equiv$ $\left(R_{C, \mathrm{MSR}}^{+}\right)^{-1}\left(\mathbb{L}^{\{1 f\}}\right)^{\frac{1}{v}} \equiv\left(R_{\xi, \mathrm{MSR}}^{+}\right)^{-\frac{1}{v}}\left(\mathbb{L}^{\{1 f\}}\right)^{\frac{1}{v}}$, we obtain $t_{0} \vartheta \propto\left(\frac{\mathbb{L}^{\{1 f\}}}{\mu}\right)^{-\frac{1}{v}}$. The latter result confirms the $t_{0}$ versus $\vartheta^{-1}$ asymptotic link, while $\mu$ well appears as the non-classical, metric-like non-universal parameter readily Ising-like similar to $\psi_{\rho}$ for the one-component fluid subclass. We also stress the pivotal scaling role played by the reference lengths, here underlined by the term $\left(\frac{\mathbb{L}^{\{1 f\}}}{\mu}\right)^{-\frac{1}{v}}$ in the $t_{0}$ versus $\vartheta^{-1}$ link. De facto, the hyperscaling-like universal combinations $\left(R_{\xi}^{+}\right)^{d}=A^{+}\left(\xi^{+}\right)^{d}$ and $Q_{c}=\left(\xi^{+}\right)^{d} \frac{B^{2}}{\Gamma^{+}}$(then $R_{C}=A^{+} \frac{\Gamma^{+}}{B^{2}}$ ), are well accounted for since $A^{+}=Z_{C, \mathrm{MSR}}^{+} \mu^{3}\left(t_{0}\right)^{\alpha-2} \equiv \mathbb{Z}_{C}^{+} \vartheta^{2-\alpha}\left(\mathbb{L}^{\{1 f\}}\right)^{d}$ and $\frac{\xi_{0, \mathrm{MSR}}^{+}}{l_{0}}=$ $Z_{\xi, \mathrm{MSR}}^{+} \mu^{-1}\left(t_{0}\right)^{v} \equiv\left(\mathbb{Z}_{\xi}^{+}\right)^{-1} \vartheta^{-v}\left(\mathbb{L}^{\{1 f\}}\right)^{-1}=\xi^{+}$, with $Z_{C, \mathrm{MSR}}^{+}=\frac{1}{16 \pi}, Z_{\xi, \mathrm{MSR}}^{+} \simeq 1.9$, $\left(R_{C}\right)_{\mathrm{MSR}}=0.0580 \approx 0.0574=\left(R_{C}\right)_{\mathrm{MR}},\left(R_{\xi}^{+}\right)_{\mathrm{MSR}}^{d}=0.0206 \approx 0.0196=\left(R_{\xi}^{+}\right)_{\mathrm{MR}}^{d}$, and $\left(Q_{c}\right)_{\mathrm{MSR}}^{-1}=2.82 \approx 2.93=\left(Q_{c}\right)_{\mathrm{MR}}^{-1}$ (see lines $5,6 \& 10$ to 12 in corresponding columns 5 $\& 7$ of Table 2).

Finally, choosing a prefixed $u$ value close to $u^{*}$ for consistency with the MSR approximations and $\{\mu, a\}$ as free, independent fitting parameters, induces the quasi-complete similarity between the MSR- $\{u ; \mu ; a\}$ - and the MR- $\left\{\vartheta ; g_{0} ; \psi_{\rho}\right\}$-non-universal parameters (with $\vartheta$ and $\psi_{\rho}$ independent). For the remaining slight differences between the estimated universal values of the exponents and amplitude combinations in each model, see lines 7 to 13, columns 5 and 7, of Table 2. The MSR versus MR matching infered from their respective non-universal sets $\{\mu ; a\}$ and $\left\{\vartheta ; \psi_{\rho}\right\}$ extracted at finite distance from $T_{c}$, confirms the Ising-like critical nature of the xenon crossover descriptions based on the renormalization methods with infinite cutoff wave number, without any reference to the parametrization of the mean field regime. Now, 
the MSR modeling can be applied to any one-component fluid whose generalized critical coordinates of liquid-gas critical point are known, since the unequivocal links $\left\{\vartheta ; \psi_{\rho}\right\}$ versus $\left\{Y_{c} ; Z_{c}\right\}$ are already given by Eqs. (19) and (20) in Sect. 2.

\section{Appendix 2.2: CLM and CPM Versus MR}

The results of the CLMs or CPM fitting cases are not basically comparable to the MR ones due to a possible, positive non-zero value of the crossover parameter $\bar{u}$ in the construction of the crossover function $Y$ (using the notation of Refs. [25] and [28]). Consequently, from the corresponding four-parameter fittings, the extraction of the true values of the two-scale-factors which are non-universal in the Ising-like asymptotic description close to the non-trivial fixed point remains unclear. Indeed, on a theoretical renormalization group approach where it is assumed that only a single irrelevant field contributes to the first-order confluent corrections to scaling, the first-order amplitudes of the confluent corrections are mandatory linked to the second crossover parameter $g$ or $\bar{u} \Lambda$, which acts as an effective Ginzburg number, while the renormalization of the mean-field-like non-universal parameters needs to only account for two suplementary non-universal bare parameters. For instance, in CPM, the introduction of the free parameters $l_{0}$ and $m_{0}$ to characterize the leading amplitudes is only due to the Ising-like universal features intrinsic to the parametric form of the scaled equation-of-state associated to the Ginzburg-number-like nature of the temperature scaling parameter $g$. Hence, the two-scale factor universality can correctly be accounted for by using the three- $\left\{g ; \widetilde{l}_{0} ; \widetilde{m}_{0}\right\}$-parameter description, as well as the two- $\left\{l_{0} ; m_{0}\right\}$ - parameter description, through the $\widetilde{l}_{0}=l_{0} g^{\beta \delta-\frac{3}{2}}$ and $\widetilde{m}_{0}=m_{0} g^{\beta-\frac{1}{2}}$ parameter rescaling. Similarly, considering the prefactor sets $\left\{\chi_{0, \mathrm{~W}}^{+}, M_{0, \mathrm{~W}}\right\}$ given in lines 6 and 7 for the model versions in columns 3 to 5 of Table 3, the comparison of the multi-parameter combinations involved in the matching equations, show that the two mean-field-like bare quantities, namely $\left\{a_{0} ; u_{0}\right\},\left\{c_{t} ; c_{\rho}\right\}$, and $\left\{\tilde{l}_{0} ; \tilde{m}_{0}\right\}$, are Ising-like renormalized only using the crossover parameter $g$ (or $\bar{u} \Lambda$ ), and not the $(1-\bar{u})$ quantity. That is formally noticeable in the asymptotical CLM-II version where are used the renormalized variables (here with subscript II) $t_{\mathrm{II}}=c_{t} \Delta \tau^{*}$ and $M_{\mathrm{II}}=c_{\rho} \Delta \tilde{\rho}$ (ignoring in xenon case the non-symmetrical behavior of the order-parameter density $\phi$, with $\phi \equiv \Delta \tilde{\rho}$ for simple fluids). In such a specific version, the respective parameter links

$$
\begin{aligned}
c_{t} & =k_{c_{t}} \vartheta(\bar{u} \Lambda)^{-\frac{1}{v}-2}=\left(A_{\mathrm{CLM}-\mathrm{II}}^{\vartheta}\right)^{-1} \vartheta(\bar{u} \Lambda)^{-\frac{1}{v}-2} \\
c_{\rho} & =k_{c_{\rho}}\left(\psi_{\rho}\right)^{-1}(\bar{u} \Lambda)^{-\frac{\eta}{2}}=A_{\mathrm{CLM}-\mathrm{II}}^{\psi_{\rho}}\left(\psi_{\rho}\right)^{-1}(\bar{u} \Lambda)^{-\frac{\eta}{2}}
\end{aligned}
$$

(see lines $12 \mathrm{~b}$ and $12 \mathrm{c}$, column 3 in Table 4 ) are obvious due to $t=\vartheta \Delta \tau^{*}$ and $m_{\mathrm{th}}=$ $\left(\mathbb{L}^{\{1 f\}}\right)^{-d}\left(\psi_{\rho}\right)^{-1} \Delta \tilde{\rho}$ in MR case. In Eqs. (68) and (69) the implicit scaling role of $\mathbb{L}^{\{1 f\}}$ is inserted in the universal prefactors

$$
\begin{aligned}
& k_{c_{t}}=\left(A_{\text {CLM-II }}^{\vartheta}\right)^{-1} \\
& k_{c_{\rho}}=A_{\text {CLM-II }}^{\psi_{\rho}}
\end{aligned}
$$

through Eqs. (54) and (55) [see also below Eqs. (72) and (73)]. The fact that the temperature scaling factor $\Delta \tau_{\mathrm{CLM}-\mathrm{II}}^{*}$ explicitly contains the product $\bar{u} \Lambda$, leads to the corresponding power-law terms $(\bar{u} \Lambda)^{\frac{1}{v}-2}$ and $(\bar{u} \Lambda)^{-\frac{\eta}{2}}$ in Eqs. (68) and (69). The Ising-like renormalization of the mean-field-like non-universal parameters $c_{t}$ and $c_{\rho}$ is only governed by $\bar{u} \Lambda$ (as the Ising-like critical limit for the crossover function $Y$, only needs the knowledge of the product $\bar{u} \Lambda$, not separately $\bar{u}$ ). Such a pivotal role of $\bar{u} \Lambda$ already mentionned in 2000 
[42], can be evidenced here by extending the previous estimations of the prefactor sets $\left\{\chi_{n, \mathrm{CLM}-\mathrm{II}}^{+}, M_{n, \mathrm{CLM}-\mathrm{II}}\right\}$ [see Eqs. (58) and (61)] to the cases of $\left\{C_{n, \mathrm{CLM}-\mathrm{II}}^{+}, \xi_{n, \mathrm{CLM}-\mathrm{II}}^{+}\right\}$for the specific heat and correlation length above $T_{c}$, where $C_{n, \mathrm{CLM}-\mathrm{II}}^{+}=(\bar{u} \Lambda)^{d}(1-\bar{u})^{\frac{\alpha-2}{\Delta}}$, and $\xi_{n, \text { CLM-II }}^{+}=(\bar{u} \Lambda)^{-1}(1-\bar{u})^{\frac{\nu}{\Delta}}$. As a remarkable result, these two correlated (since $\left.1 \equiv C_{n, \mathrm{CLM}-\mathrm{II}}^{+}\left(\xi_{n, \mathrm{CLM}-\mathrm{II}}^{+}\right)^{d}\right)$ asymptotic prefactors are non-dependent on the non-universal parameters $c_{t}$ and $c_{\rho}$. The respective comparisons with $C_{r}^{+}=\left(\mathbb{L}^{\{1 f\}}\right)^{d}$ and $\xi_{r}^{+}=\left(\mathbb{L}^{\{1 f\}}\right)^{-1}$, non-dependent of $\vartheta$ and $\psi_{\rho}$ in the case of MR, in order to match $A^{+}$and $\xi^{+}$, implie $\frac{\mathbb{L}^{\{1 f\}}}{\bar{u} \Lambda}(1-\bar{u})^{\frac{v}{\Delta}}=R_{\xi, \text { CLM-II }}^{+}=\left(R_{C, \mathrm{CLM}-\mathrm{II}}^{+}\right)^{-\frac{1}{d}} \cdot R_{\xi, \mathrm{CLM}-\mathrm{II}}^{+}$and $R_{C, \mathrm{CLM}-\mathrm{II}}^{+}$are the CLM-II versions of the universal ratios $R_{C, \mathrm{M}}^{+}$and $R_{\xi, \mathrm{M}}^{+}$previously introduced in Appendix 2.1. As for $\left(\frac{\mathbb{L}^{\{1 f\}}}{\mu}\right)^{-\frac{1}{v}}$ in the case of MSR, the concomitant pivotal roles of $\left(\mathbb{L}^{\{1 f\}}\right)^{-\frac{1}{v}}$ and $(\bar{u} \Lambda)^{-\frac{1}{v}}$ in the case of CLM-II, infer (anticipating the discussion below) the scaling length nature of the constraint reported in line 9, column 3 in Table 4, since $\left(\mathbb{L}^{\{1 f\}}\right)^{-\frac{1}{v}}=L_{\text {CLM-II }}^{\vartheta}\left(R_{\xi, \text { CLM-II }}^{+}\right)^{-\frac{1}{v}}=$ $L_{\text {CLM-II }}^{\vartheta}\left(R_{C, \text { CLM-II }}^{+}\right)^{\frac{1}{2-\alpha}}\left(\right.$ with $\left.1 \equiv R_{C, \text { CLM-II }}^{+}\left(R_{\xi, \text { CLM-II }}^{+}\right)^{d}\right)$.

Such a Ising-like renormalization of the bare parameters only using $\bar{u} \Lambda$ or $g$ is implicitly contained in all the crossover model versions [25], including CPM [27,28], based on simplest, similar, three-term mean-field-like Hamiltonians (including a square-gradient term), but the related data fitting validation needs to mandatorily be performed within the PAD. Unfortunately, as already mentioned, the absence of experimental data within the PAD prevents de facto any accurate independent estimation of the free sets $\left\{a_{0} ; u_{0}\right\},\left\{c_{t} ; c_{\rho}\right\}$, or $\left\{l_{0} ; m_{0}\right\}$. When the data fitting is performed beyond the PAD, if a single value of $\Delta \tau_{\mathrm{W}}^{*}$ exists, then the correlated prefactor set which can be extracted from the fit is not $\left\{\chi_{0, \mathrm{~W}}^{+}, M_{0, \mathrm{~W}}\right\}$, but $\left\{\chi_{n, \mathrm{~W}}^{+}, M_{n, \mathrm{~W}}\right\}$ where we have already noted that each readily independent prefactor shows a remaining $(1-\bar{u})$ dependence. As an unavoidable result, the two non-universal parameters that characterize the asymptotic two-scale-factor universality of the fluid are always dependent on the two crossover parameters in such data fittings using four adjustable parameters at a finite distance from $T_{c}$.

Consequently, CLMs or CPM joint fits of the susceptibility and coexisting density data always contain four non-universal parameters for only three resulting independent values of the free multi-parameter combinations involved in the PAD description conforms to the one calculated along the renormalized trajectory. Fitting of any supplementary singular property measured at finite distance from $T_{c}$ along the critical isochore, do not change the situation since the universal features of the $O(1)$ universality class are intrinsically accounted for in the construction of these parametric models. That makes it necessary to introduce an arbitrary character in the practical use of these parametric models, as for instance, when was used by the authors [28] the fixed value $\frac{\Lambda}{\left(c_{t}\right)^{\frac{1}{2}}}=\pi$ in their fits of the singular behaviors of ${ }^{3} \mathrm{He}$ properties. The same arbitrary value was also assumed in Ref. [13] for the CPM fitting data (with comparable values for the asymptotic multiparameter combinations given in lines 6 and 7 of Table 3) and for the estimation of the first-order confluent corrections in the case of CMM to obtain ideal match with MR without adjustable parameter. Such arbitrariness cannot be suppressed due to the basic construction of the mean-field limit $Y \rightarrow 1$ for the crossover function $Y$, using separately $\bar{u}$ and $\bar{u} \Lambda$. 
According to the above situation, we must now account for a single common $\vartheta$ value that governs the joint matching of the leading and the confluent amplitudes of the PAD description, i.e., $(\vartheta)_{1}=(\vartheta)_{0} \equiv \vartheta$ in Eqs. (53) and (56). The resulting constrained multiparameter combinations $L_{\mathrm{M}}^{\vartheta}=\frac{A_{\mathrm{M}}^{\vartheta}}{R_{\mathrm{M}}^{1}}$ (see line 9 of Table 4 ) never limit a possible continuous change of the multiplicative term $(1-\bar{u})^{\frac{1}{\Delta}}$ with $\bar{u} \geq 0$. This change can thus be compensated by the correlated continuous change of the product $\bar{u} \Lambda$, which limits the capability of proof that a single value for the temperature scaling factor $\Delta \tau_{\mathrm{M}}^{*}$ exists. Such implicit correlations between these three quantities are also easy to understand with the CLM-II parameter set $\left\{\Lambda ; \bar{u} ; c_{t} ; c_{\rho}\right\}$, where the ratio $\frac{1-\bar{u}}{L_{\mathrm{M}}^{\vartheta}}=\bar{u} \Lambda$ remains free after the constraint $(\vartheta)_{1}=(\vartheta)_{0} \equiv \vartheta$ (see line 10, column 4 in Table 4). An accurate illustration was already shown in Ref. [42,43] using the explicit correlations between $Y_{c}$ and $Z_{c}$ and the CLM-II fitting results $\left\{c_{t} ; c_{\rho}\right\}$ obtained for six differents simple fluids. A similar view can be given on the large field of the theoretical studies focussed on the modeling of different critical-to-classical crossovers. Indeed, it should also be noted the case of an asymptotic version of CLM [41] used through an explicit comparison with the numerical critical-to-classical crossover calculations in Ising lattice models of different interaction ranges, via a tunable range $R$ of interactions [30]. Such a particular asymptotic CLM modeling via a tunable $\bar{u}$ [45] leads to the recovery of common mean-field limits for $\bar{u} \rightarrow 0$ and $R \rightarrow \infty$, while introducing phenomenological arguments to define the practical continuous adjustements of the $\bar{u}(R) \sim \frac{1}{R^{4}}$ dependence at small $R$ to account for finite-sized effects up to the limit $R=1$. Therefore, in the absence of similar additional theoretical arguments able to link (at least) one crossover parameter to the wellknown short-ranged interactions between xenon particles, such four free parameters of the CLMs or CPM crossover fittings remain not easy to compare with the three non-universal parameters of the MR crossover functions. Nevertheless, the matching of the CLM-I\&II and CPM PAD description to the MR one can partly be formulated through the universale multiparameter combinations given respectively in lines 9 and 10, columns 3 to 5 of Table 4. For example, from a generalization of Eqs (68) and (69) to the three models, the different prefactors $k_{i}$, where the subscript $i$ refers to the selected non-universal parameter of the model, are universal quantities still depending on $\mathbb{L}^{\{1 f\}}$ and the universal ratios of lines 1 to 3. As mentioned by the quantity noted \{\}$^{\star}$ in subline 9 , column 5 , only the addition of an arbitrary constraint, such as $\frac{\Lambda}{\left(c_{t}\right)^{\frac{1}{2}}}=\pi$, closes the unequivocal link between the respective non-universal (reduced) parameter set $\left\{\bar{u} ; l_{0} ; m_{0}\right\}$ of CPM versus the one $\left\{\vartheta ; g_{0} ; \psi_{\rho}\right\}$ of MR (see also Table 3).

\section{Appendix 2.3: CLM0 and CPMO Versus MR via MSR}

On the basis of the present work, it is noticeable that the PAD matching can be formally constructed by fixing $\bar{u}=0$ in the above model versions. CLM0-I\&II and CPM0 thus appear to be conform to the infinite cutoff wavenumber $\Delta \rightarrow \infty$ and a finite value of $\bar{u} \Lambda$. From lines 8 or 9 with $\bar{u}=0$, the constrained subparameters $g \propto \vartheta^{-1}$ or $\bar{u} \Lambda$ (fixed) given in line 11 of Table 4 can be derived in a straightforward manner. Now, the closed unequivocal link between the respective non-universal parameter sets of the W versus MR comparison is obtained through the equations given respectively in lines $12 \mathrm{a}, 12 \mathrm{~b}$ and $12 \mathrm{c}$, columns 3 to 5 of Table 4, where line 12a replaces previous line 9 . Here is mentioned the $\bar{u} \Lambda$ quantity noted \{\}$^{\star}$ in line 12 , column 4 , which is fixed in the case of CLM0-II. The universal prefactors are defined in terms of the universal ratios given in Table 4, as follows 


$$
\begin{aligned}
& k_{a_{0}}=\frac{\left(R_{\mathrm{CLM}-\mathrm{I}}^{1,+}\right)^{\gamma-1}}{R_{\chi, \mathrm{CLM}-\mathrm{I}}^{+}}\left(\mathbb{L}^{\{1 f\}}\right)^{d} ; k_{c_{t}}=\left(A_{\mathrm{CLM}-\mathrm{II}}^{\vartheta}\right)^{-1} ; k_{l_{0}}=\frac{R_{M, \mathrm{CPM}}}{R_{\chi, \mathrm{CPM}}^{+}} \\
& k_{u_{0}}=\frac{\left(R_{\mathrm{CLM}-\mathrm{I}}^{1,+}\right)^{\gamma-2 \beta}}{R_{\chi, \mathrm{CLM}-\mathrm{I}}^{+}\left(R_{M, \mathrm{CLM}-\mathrm{I}}\right)^{2}}\left(\mathbb{L}^{\{1 f\}}\right)^{-3 d} ; k_{c_{\rho}}=A_{\mathrm{CLM}-\mathrm{II}}^{\psi_{\rho}} ; k_{m_{0}}=R_{M, \mathrm{CPM}}\left(\mathbb{L}^{\{1 f\}}\right)^{d}
\end{aligned}
$$

The above model matching now enlarges the physical understanding of the non-universal parameters. For instance, from the MSR versus CPM0 comparison, $t_{0}=\frac{\mu^{2}}{a}$ and $g=\frac{(\bar{u} \Lambda)^{2}}{c_{t}}$ take similar nature of temperature scaling factors, which confers a strict functional equivalence between the dimensionless length scaling parameters $\mu$ and $\bar{u} \Lambda$ on one hand, and the dimensionless temperature scaling parameters $a$ and $c_{t}$ on the other. Therefore, in a first step, we can assume $g_{\chi, \mathrm{MSR}}^{1,+}=g_{\chi, \mathrm{CPM}}^{1,+}$, taking advantage of the small differences between the universal values $g_{\chi, \mathrm{MSR}}^{1,+}=0.545$ of SRM and $g_{\chi}^{1,+},+{ }_{\text {CPM }}=0.590$ of CPM (see line 1, columns 4 and 7 , in Table 2). Hence, the confluent amplitude matching between $a_{\chi}^{+}$of both models implies $g=t_{0}\left(1-\frac{u}{u^{*}}\right)^{-\frac{1}{\Delta}}$. Introducing thus $\mu=\mu_{\mathrm{MSR}}^{*}\left(1-\frac{u}{u^{*}}\right)^{\frac{v}{\Delta}}, a=a_{\mathrm{MSR}}^{*}\left(1-\frac{u}{u^{*}}\right)^{\frac{2 v-1}{\Delta}}$, with resulting $t_{0}=\frac{\left(\mu_{\mathrm{MSR}}^{*}\right)^{2}}{a_{\mathrm{MSR}}^{*}}\left(1-\frac{u}{u^{*}}\right)^{\frac{1}{\Delta}}$ for the case of MSR, finally leads to $g=\frac{\left(\mu_{\mathrm{MSR}}^{*}\right)^{2}}{a_{\mathrm{MSR}}^{*}}$ for the case of CPM0. The bare parameters $\mu_{\mathrm{MSR}}^{*}$ and $a_{\mathrm{MSR}}^{*}$ are only defined for the fixed point limit $u=u^{*}$, i.e., without any reference to the mean-field regime. Similarly, the asymptotical Ising-like matching between $B$ and $\Gamma^{+}$values estimated on CPM0 and MSR provides $\widetilde{m}_{0}=\frac{Z_{M, \text { SRM }}}{Z_{M, \mathrm{CPM}}}\left(\frac{a_{\mathrm{MSR}}^{*}}{\mu_{\mathrm{MSR}}^{*}}\right)^{\frac{1}{2}}\left(1-\frac{u}{u^{*}}\right)^{\frac{v-2 \beta}{2 \Delta}}$ and $\widetilde{l}_{0}=\frac{Z_{\chi, \mathrm{CPM}}}{Z_{\chi, \mathrm{SRM}}} \frac{Z_{M, \mathrm{SRM}}}{Z_{M, \mathrm{CPM}}}\left(\frac{a_{\mathrm{MSR}}^{*}}{\mu_{\mathrm{MSR}}^{*}}\right)^{\frac{1}{2}} a_{0}\left(1-\frac{u}{u^{*}}\right)^{\frac{2 \beta-v}{2 \Delta}}$, which closes the expected non-analytic links between the MSR- $\{u ; \mu ; a\}$ non-universal parameters and the CPM0- $\left\{g ; \widetilde{l}_{0} ; \widetilde{m}_{0}\right\}$ ones and simultaneously reduces by one the number of independent non-universal parameters. The results of the MSR data fittings with a prefixed $u$ value close to $u^{*}$, with $\mu$ and $a$ as free fitting independent parameters, are then strictly similar to the ones of the CPM0 data fittings with a prefixed $\bar{u}=0$ value and $g, \widetilde{m}_{0}$, and $\widetilde{l}_{0}$ as free fitting parameters. Subsequently, $m_{0}=\widetilde{m}_{0} g^{\frac{1}{2}-\beta}$ and $l_{0}=\widetilde{l}_{0} g^{\frac{3}{2}-\beta \delta}$ come out as being the two Ising-like independent, non-universal parameters of CPM0, similar to the two Ising-like independent, non-universal parameters $\mu$ and $a$ of SRM. Our previous comparison between (mean and/or master) MR and SRM fitting results extends the similarity (in number and physical nature) to the three MR- $\left\{\vartheta ; g_{0} ; \psi_{\rho}\right\}$, and/or master $-\left\{Y_{c} ; \alpha_{c} ; Z_{c}\right\}$ parameter sets (with $\mathbb{L}^{\{1 f\}}=g_{0} \alpha_{c}$ ), where the two Ising-like independent, non-universal scale factors are $\vartheta$ and $\psi_{\rho}$ (and/or $Y_{c}$ and $Z_{c}$ ). The next step, not examined here, will focus on the development of better matching methods in order to account for the small differences between $g_{\chi, \text { MSR }}^{1,+}$ and $g_{\chi}^{1,+}+\mathrm{CPM}$. In such a future work, it will also become important to integrate the reduction of diffences between the universal values of the critical exponents and the amplitude combinations, using an upgraded version of CMM which matches as well as possible the universal estimates from the MR scheme.

\section{References}

1. Garrabos, Y., Lecoutre, C., Marre, S., LeNeindre, B.: Critical crossover functions for simple fluids: nonanalytical scaling determination of the Ising-like crossover parameter. J. Stat. Phys. 164, 575-615 (2016) 
2. Bagnuls, C., Bervillier, C.: Classical-to-critical crossovers from field theory. Phys. Rev. E 65, 066132-12p (2002). And references therein

3. Garrabos, Y., Bervillier, C.: Mean crossover functions for uniaxial 3D ising-like systems. Phys. Rev. E 74, 021113-16p (2006)

4. Zinn-Justin, J.: Quantum Field Theory and Critical Phenomena, 4th edn. Oxford University Press, Oxford (2002)

5. Pelissetto, A., Vicari, E.: Critical phenomena and renormalization group theory. Phys. Rep. 368, 549-727 (2002)

6. Wilson, K.G., Kogut, J.: The renormalization group and the $\varepsilon$ expansion. Phys. Rep. 12C, 75-200 (1974)

7. Privman, V., Hohenberg, P.C., Aharony, A.: Universal critical point amplitude relations. In: Domb, C., Lebowitz, J.B. (eds.) Phase Transitions and Critical Phenomena, vol. 14, pp. 1-134. Academic Press, New York (1991)

8. Guida, R., Zinn-Justin, J.: Critical exponents of the N-vector model. J. Phys. A 31, 8103-8122 (1998)

9. Garrabos, Y., Lecoutre-Chabot, C., Palencia, F., LeNeindre, B., Erkey, C.J.: Master crossover functions for one-component fluids. Phys. Rev. E. 77, 021116-26p (2008)

10. Garrabos, Y.: Contribution à l'étude des propriétés d'état des fluides purs dans leur région critique, Thèse de Doctorat d'état, Université Paris 6 (1982)

11. Garrabos, Y.: Facteurs d'échelle phénoménologiques pour la transition critique liquide-gaz des fluides purs. J. Phys. 46, 281 (1985). For an english version see also: Phenomenological Scale Factors for the Liquid-Vapor Critical Transition of Pure Fluids, cond-mat/0512408

12. Garrabos, Y.: Scaling behaviour of the fluid subclass near the liquid-gas critical point. J. Phys. 47, 197-206 (1986)

13. Garrabos, Y., Lecoutre, C., Marre, S., Guillaument, R., Beysens, D., Hahn, I.: Crossover equation of state models applied to the critical behavior of xenon. J. Stat. Phys. 158, 1379-1412 (2015)

14. Güttinger, H., Cannell, D.S.: Corrections to scaling in the susceptibility of xenon. Phys. Rev. A 24, 3188-3201 (1981)

15. Närger, U., Balzarini, D.A.: Coexistence-curve diameter and critical density of xenon. Phys. Rev. B 42, 6651-6657 (1990)

16. Bagnuls, C., Bervillier, C.: Nonasymptotic critical behaviour from field theory for Ising like systems in the homogeneous phase: theoretical framework. J. Phys. Lett. 45, L95-L100 (1984)

17. Bagnuls, C., Bervillier, C.: Classical-to-critical crossovers from field theory. Phys. Rev. B 32, 7209 (1985)

18. Bagnuls, C., Bervillier, C., Garrabos, Y.: Experimental data analysis on xenon above the critical temperature from nonlinear renormalization group. J. Phys. Lett. 45, L127-L132 (1984)

19. Schloms, R., Dohm, V.: Minimal renormalization without $\varepsilon$-expansion: critical behavior in three dimensions. Nucl. Phys. B 328, 639-663 (1989)

20. Krause, H.J., Schloms, R., Dohm, V.: Minimal renormalization without $\varepsilon$-expansion: amplitude functions in three dimensions. Z. Phys. B 79, 287-293 (1990)

21. Hahn, I., Zhong, F., Barmatz, M., Haussmann, R., Rudnick, J.: Crossover behavior in the isothermal susceptibility near the 3 He critical point. Phys. Rev. E 63, 055104(R)-4p (2001)

22. Zhong, F., Barmatz, M., Hahn, I.: Application of minimal substraction renormalization to crossover behavior near the 3He liquid-vapor critical point. Phys. Rev. E 67, 021106-20p (2003)

23. Chen, Z.Y., Albright, P.C., Sengers, J.V.: Crossover from singular critical to regular classical thermodynamic behavior of fluids. Phys. Rev. A 41, 3161-3177 (1990)

24. Chen, Z.Y., Abbaci, A., Tang, S., Sengers, J.V.: Global thermodynamic behavior of fluids in the critical region. Phys. Rev. A 42, 4470-4484 (1990)

25. Tang, S., Sengers, J.V., Chen, Z.Y.: Nonasymptitic critical thermodynamical behavior of fluids. Physica A 179, 344-377 (1991)

26. Anisimov, M.A., Povodyrev, A.A., Kulikov, V.D., Sengers, J.V.: Nature of crossover between ising-like and mean-field critical behavior in fluids and fluid mixtures. Phys. Rev. Lett. 75, 3146-3149 (1995)

27. Agayan, V.A., Anisimov, M.A., Sengers, J.V.: A crossover parametric equation of state for threedimensional Ising universality class systems, In: Proceedings of 14th Symposium on Thermophysical Properties, Boulder, Colorado, June 25-30 (2000)

28. Agayan, V.A., Anisimov, M.A., Sengers, J.V.: Crossover parametric equation of state for Ising-like systems. Phys. Rev. E 64, 026125 (2001)

29. Luijten, E., Binder, K.: Nature of crossover from classical to Ising-like critical behavior, Phys. Rev. E 58, R4060-R3261 (1998); Erratum, Phys. Rev. E 59, 7254(E) (1999)

30. Luijten, E.: Critical properties of the three-dimensional equivalent-neighbor model and crossover scaling in finite systems. Phys. Rev. E 59, 4997-5008 (1999)

31. Luijten, E., Meyer, H.: Crossover behavior in $3 \mathrm{He}$ and Xe near their liquid-vapor critical point. Phys. Rev. E 62, 3257-3261 (2000) 
32. Ginzburg, V.L.: Phase transitions of the second kind and the microscopic theory of ferroelectricity, Fiz. Tverd. Tela 2, 2031-2043 (1960) [Sov. Phys. Solid State 2, 1824-1836 (1961)

33. Pelissetto, A., Rossi, P., Vicari, E.: Crossover scaling from classical to nonclassical critical behavior. Phys. Rev. E 58, 7146-7150 (1998)

34. Wegner, F.J.: Corrections to scaling laws. Phys Rev. B 5, 4529-4536 (1972)

35. Nicoll, J.F., Bhattacharjee, J.K.: Crossover functions by renormalization-group matching: $\mathrm{O}\left(\varepsilon^{2}\right)$ results. Phys. Rev. B 23, 389-401 (1981)

36. Nicoll, J.F., Albright, P.C.: Crossover functions by renormalization-group matching: three-loop results. Phys. Rev. B 31, 4576-4589 (1985)

37. Nicoll, J.F., Albright, P.C.: Background fluctuations and Wegner corrections. Phys. Rev. B 34, 1991-1996 (1986)

38. Albright, P.C., Sengers, J.V., Nicoll, J.F., Ley-Koo, M.: A crossover description for the thermodynamic properties of fluids in the critical region. Int. J. Thermophys. 7, 75-85 (1986)

39. Albright, P.C., Chen, Z.Y., Sengers, J.V.: Crossover from singular to regular thermodynamic behavior of fluids in the critical region. Phys. Rev. B 36, 877-880 (1987)

40. Belyakov, M.Y., Kiselev, S.B.: Crossover behavior of the susceptibility and the specific heat near a second-order phase transition. Physica A 190, 75-94 (1992)

41. Anisimov, M.A., Kiselev, S.B., Sengers, J.V., Tang, S.: Crossover approach to global critical phenomena in fluids. Physica A 188, 487-525 (1992)

42. Garrabos, Y., Le Neindre, B., Wunenburger, R., Lecoutre-Chabot, C., Beysens, D.: Universal scaling form of the equation of state of a critical pure fluid. In: Proceedings of 14th Symposium on Thermophysical Properties, Boulder, Colorado, June 25-30 (2000)

43. Garrabos, Y., Le Neindre, B., Wunenburger, R., Lecoutre-Chabot, C., Beysens, D.: Universal scaling form of the equation of state of a critical pure fluid. Int. J. Thermophys. 23, 997-1011 (2002)

44. Zhong, F., Barmatz, M.: Comparison of theoretical models of crossover behavior near the 3He liquid-vapor critical point. Phys. Rev. E 70, 066105-9p (2004)

45. Kim, Y.C., Anisimov, M.A., Sengers, J.V., Luijten, E.: Crossover critical behavior in the three-dimensional Ising model. J. Stat. Phys. 110, 591-609 (2003)

46. Michels, A., Wassenaar, T., Louwerse, P.: Isotherms of xenon at temperatures between $0^{\circ} \mathrm{C}$ and $150^{\circ} \mathrm{C}$ and at densities up to 515 Amagats (pressures up to 2800 atmospheres). Physica 20, 99-106 (1954)

47. Lecoutre, C., Guillaument, R., Marre, S., Garrabos, Y., Beysens, D., Hahn, I.: Weightless experiments to probe universality of fluid critical behavior. Phys. Rev. E 91, 060101(R)-5p (2015)

48. Edwards, C., Lipa, J.A., Buckingham, M.J.: Specific heat of xenon near the critical point. Phys. Rev. Lett. 25, 496-499 (1968)

49. Wilcox, L.R., Balzarini, D.: Interferometric determination of near-critical isotherms of xenon in the earth's field. J. Chem. Phys. 48, 753-763 (1968)

50. Estler, W.T., Hocken, R., Charlton, T., Wilcox, L.R.: Coexistence curve, compressibility, and the equation of state of xenon near the critical point. Phys. Rev. A 12, 2118-2136 (1975)

51. Hocken, R.J., Moldover, M.R.: Ising critical exponents in real fluids: an experiment. Phys. Rev. Lett. 37, 29-32 (1976)

52. Sengers, J.V., Moldover, M.R.: Two-scale-factor universality near the critical point of fluids. Phys. Lett. 66A, 44-46 (1978)

53. Fisher, M.E., Zinn, S.-Y., Hupton, P.J.: Trigonometric models for scaling behavior near criticality. Phys. Rev. B 59, 14533-14545 (1999)

54. Wilcox, L.R., Estler, W.T.: On the phenomenology of phase transitions. J. Phys. Colloq. 32, C5a-175 (1974) 